Original paper

\title{
Quartz-apatite-REE phosphates-uraninite vein mineralization near Čučma (eastern Slovakia): a product of early Alpine hydrothermal activity in the Gemeric Superunit, Western Carpathians
}

\author{
Martin ŠTEVKO*, Pavel UHER, Martin ONDREJKA, Daniel OZDÍN, Peter BAČíK
}

Department of Mineralogy and Petrology, Faculty of Natural Sciences, Comenius University, Mlynská dolina G, 84215 Bratislava, Slovakia;stevko@fns.uniba.sk

* Corresponding author

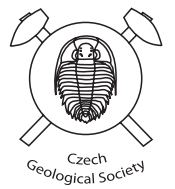

The quartz veins with primary fluorapatite, xenotime-(Y), monazite-(Ce) to monazite-(Nd), uraninite, and secondary florencite-(Ce) and goyazite occur in Lower Devonian metavolcano-sedimentary sequence of the Gelnica Group, Gemeric Superunit, the Central Western Carpathians (eastern Slovakia). They represent an example of hydrothermal REE-U mineralization. Fluorapatite forms parallel bands of columnar crystals $(\leq 3 \mathrm{~cm})$ in massive quartz. Monazite$(\mathrm{Ce})$ to $(\mathrm{Nd})$ shows a near end-member composition with very small amounts of cheralite and huttonite components. Widespread xenotime-(Y) forms colloform aggregates or irregular aggregates in association with fluorapatite and monazite. Uraninite electron-microprobe $\mathrm{U}-\mathrm{Pb}$ dating gave the average age of $207 \pm 2 \mathrm{Ma}(\mathrm{n}=16,2 \sigma)$, which is consistent with formation of the U mineralization in the Gemeric Superunit (e.g., Kurišková uranium deposit) during early Alpine hydrothermal activity.

Keywords: REE phosphates, florencite, uraninite, early Alpine activity, Čučma, Slovakia

Received: 2 September 2013; accepted: 22 May 2014; handling editor: J. Sejkora

\section{Introduction}

Rare-earth phosphate minerals occur commonly in magmatic and metamorphic rocks, both as scattered accessory minerals or REE-rich accumulations. However, later mobilization of REE under hydrothermal conditions leads to formation of various types of mineralization, enriched in monazite, rhabdophane, xenotime and other REE phosphate and carbonate phases, frequently together with U-, Th-, and Zr-rich minerals (e.g., Möller 1989; Yongliang and Yusheng 1991; Gieré 1996; Samson and Wood 2005). Such types of mineralization commonly have significant REE and Y economic potential. However, even the knowledge of small and uneconomic quartz-phosphate hydrothermal vein occurrences may improve our understanding of large REE ore deposits, as well as behavior of REE-phosphate phases in hydrothermal processes.

Quartz-apatite veins in the vicinity of Ččma village (Gemeric Superunit, eastern Slovakia) represents an example of a small, sub-economic accumulation of REE and U. However, it belongs to the most significant local accumulations of REE minerals on the territory of the Western Carpathians (Rojkovič et al. 1999). This contribution is focused on detailed mineralogical characterization of this hydrothermal REE-U mineralization, in particular of primary and secondary REE-bearing phosphate phases, as well as associated fluorapatite and uraninite. In addition, $\mathrm{U}-\mathrm{Th}-\mathrm{Pb}$ chemical dating of uraninite helps to decipher the temporal context and petrogenesis of the mineralization.

\section{Geological setting}

Hydrothermal veins with the REE-U mineralization at Čucma are hosted in the medium-, to coarse-grained rhyolite metatuffs (main vein) and laminated quartz-sericite or graphite-sericite phyllites (Majerská Valley) of the Bystrý Potok Fm. which belongs to the Gelnica Group (Bajaník ed. 1983, 1984) (Fig. 1). Other authors characterized the host rocks as greenish and grey-green porphyroblastic to gneissic biotite-muscovite metapelites, quartzites and quartzite gneisses of the Zbojnícky kameň Beds (Lower Devonian) of the Smolník Fm., the Gelnica Group, and the Volovec Supergroup (Grecula et al. 2009, 2011).

The Gelnica Group as a part of the Gemeric Superunit consists mainly of siliciclastic deep-water turbidite sequence with rhyolite/dacite volcanites and volcaniclastics and lydites, which were metamorphosed at lower greenschist-facies conditions (Snopko 1967; Ivanička et al. 1989; Faryad 1991; Vozárová 1993). It is divided to three formations, defined from the bottom upwards: the Vlachovo Fm., the Bystrý Potok Fm., and the Drnava Fm. (Bajaník ed. 1983; Ivanička et al. 1989). The assumed Early Paleozoic (Late Cambrian to Early Devonian) 


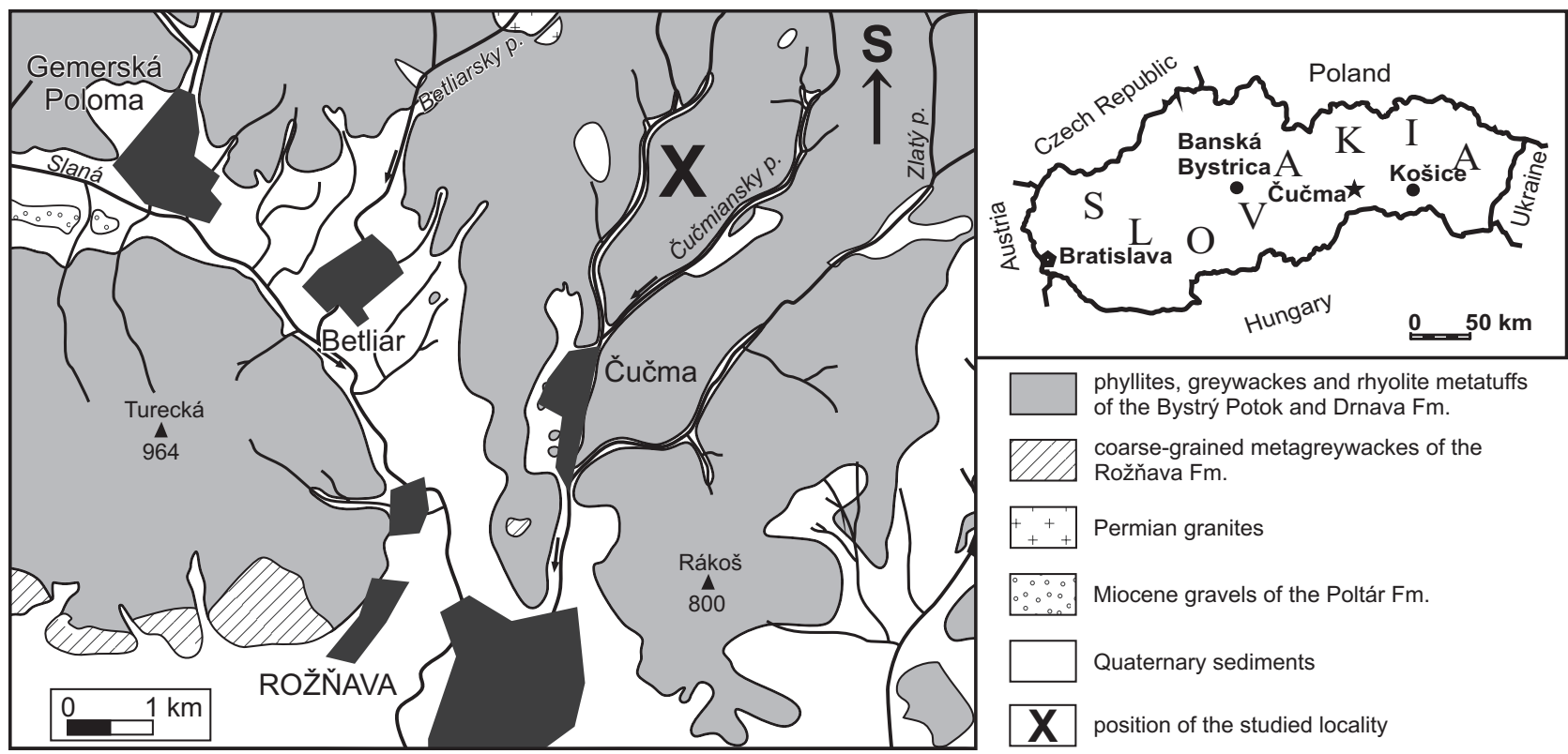

Fig. 1 Geological map of the Čučma area, Gemeric Superunit (modified after Bajaník et al. 1983).

age of the Gelnica Group, based on sporomorphs and acritarch assemblages (Snopková and Snopko 1979) and agglutinated foraminifers (Vozárová et al. 1998; Soták et al. 1999), was recently constrained by U-Th-Pb SHRIMP dating on zircons from acid volcanic rocks (Putiš et al. 2008; Vozárová et al. 2010). Concordant magmatic zircons from the metavolcanic rocks of the Bystrý Potok $\mathrm{Fm}$. gave an average $\mathrm{U}-\mathrm{Pb}$ age of $465.8 \pm 1.5 \mathrm{Ma}$ (Vozárová et al. 2010).

The outcrop of the main quartz-apatite vein associated with the REE-U mineralization is located $2.5 \mathrm{~km}$ north of the Čučma village in the Spišsko-Gemerské Rudohorie Mts., eastern Slovakia (Fig. 1). The vein is $c$. $1.7 \mathrm{~km}$ long, up to $3 \mathrm{~m}$ thick and dips $65^{\circ}$ to SSE. Small occurrences with similar mineralization are also known from the adjacent Majerská Valley and also in the vicinity of Betliar, Helcmanovce, and Kociha villages (Šváb et al. 1966; Rojkovič et al. 1999). The occurrence was discovered and prospected by the former Uranium Survey company in 1960's (Šváb et al. 1966). First mineralogical characterization of the REE-U mineralization near Čučma was given in unpublished report of Pelymsky (Tréger 1973) who described quartz, apatite, xenotime, uraninite, and pyrite as main minerals. Later, other phosphate minerals were identified: monazite (Varček 1977) and goyazite with plumbogummite (Rojkovič 1993). Vein filling consists primary of quartz and fluorapatite with local accumulations of REE and U minerals (Tréger 1973; Varček 1977; Rojkovič et al. 1999). Among the ore minerals pyrite, arsenopyrite, chalcopyrite, galena, and molybdenite occur in small quantities (Tréger 1973; Varček 1977). Supergene minerals are represented by autunite, goethite, goyazite, opal, plumbogummite, and torbernite (Rojkovič 1993, 1997; Rojkovič et al. 1999; Uher and Števko 2009).

According to Rojkovič (1997) and Rojkovič et al. (1999), hydrothermal quartz-apatite veins with the REE$\mathrm{U}$ mineralization in the Gemeric Superunit are genetically related to the Permian granites, whereas Lower Paleozoic black phyllites and lydites of the Gelnica Group are regarded as a probable source of REE. The post-orogenic granites (the Spiš-Gemer type) belong to the Permian specialized S-type granites enriched in $\mathrm{P}, \mathrm{Li}, \mathrm{Sn}, \mathrm{W}, \mathrm{Nb}$, Ta, Th, U, F, and B (e.g., Petrík and Kohút 1997; Broska and Uher 2001; Poller et al 2002; Kohút and Stein 2005). Presence of the Spiš-Gemer granites was confirmed by exploratory drilling c. $220 \mathrm{~m}$ below the main quartz-apatite vein near Čučma (Šváb et al. 1966). Moreover, the granites occur in the nearby Gabriela adit, at a distance of up to $\sim 500 \mathrm{~m} \mathrm{NE}$ from the studied vein with REE-U mineralization. Fluid phases and thermal energy, both generated from the intrusion of these granites, were taken responsible for mobilization of $\mathrm{P}, \mathrm{REE}$, and $\mathrm{U}$ into the hydrothermal veins (Rojkovič et al. 1999).

\section{Analytical methods}

The electron-microprobe analyses (EMPA) of minerals were carried out by Cameca SX100 electron microprobe (Dionýz Štúr State Geological Institute, Bratislava) at the wave-dispersion mode (WDS) with an accelerating voltage of $15 \mathrm{kV}$, beam current of 20-100 nA and beam diameter of $1-5 \mu \mathrm{m}$. The following standards and lines were used for analyzing the studied minerals: barite $\left(\mathrm{S} K_{\alpha}\right)$, apatite $\left(\mathrm{P} K_{\alpha}\right)$, GaAs $\left(\operatorname{As} L_{\alpha}\right)$, wollastonite (Si $K_{\alpha}$, Ca $K_{\alpha}$ ), 
$\mathrm{TiO}_{2}\left(\mathrm{Ti} K_{\alpha}\right), \mathrm{ZrSiO}_{4}\left(\mathrm{Zr} L_{\alpha}\right), \mathrm{HfO}_{2}\left(\mathrm{Hf} L_{\alpha}\right), \mathrm{ThO}_{2}\left(\mathrm{Th} M_{\alpha}\right)$, $\mathrm{UO}_{2}\left(\mathrm{U}_{\beta}\right), \mathrm{Al}_{2} \mathrm{O}_{3}\left(\mathrm{Al} K_{\alpha}\right), \mathrm{ScPO}_{4}\left(\mathrm{Sc} K_{\alpha}\right), \mathrm{YPO}_{4}\left(\mathrm{Y}_{\alpha}\right)$, $\mathrm{LaPO}_{4}\left(\mathrm{La} L_{\alpha}\right), \mathrm{CePO}_{4}\left(\mathrm{Ce} L_{\alpha}\right), \mathrm{PrPO}_{4}\left(\operatorname{Pr} L_{\beta}\right), \mathrm{NdPO}_{4}$ $\left(\mathrm{Nd} L_{\alpha}\right), \mathrm{SmPO}_{4}\left(\mathrm{Sm} L_{\alpha}\right), \mathrm{EuPO}_{4}\left(\mathrm{Eu} L_{\beta}\right), \mathrm{GdPO}_{4}\left(\mathrm{Gd} L_{\alpha}\right)$, $\mathrm{TbPO}_{4}\left(\mathrm{~Tb} L_{\alpha}\right), \mathrm{DyPO}_{4}\left(\mathrm{Dy} L_{\beta}\right), \mathrm{HoPO}_{4}\left(\mathrm{Ho}_{\beta}\right), \mathrm{ErPO}_{4}$ $\left(\operatorname{Er} L_{\beta}\right), \mathrm{TmPO}_{4}\left(\mathrm{Tm} L_{\alpha}\right), \mathrm{YbPO}_{4}\left(\mathrm{Yb} L_{\alpha}\right), \mathrm{LuPO}_{4}\left(\mathrm{Lu} L_{\beta}\right)$, fayalite $\left(\mathrm{Fe} K_{\alpha}\right)$, rhodonite $\left(\mathrm{Mn} K_{\alpha}\right)$, forsterite $\left(\mathrm{Mg} K_{\alpha}\right)$, $\mathrm{SrTiO}_{3}\left(\mathrm{Sr} L_{\alpha}\right)$, barite $\left(\mathrm{Ba} L_{\alpha}\right), \mathrm{PbCO}_{3}\left(\mathrm{~Pb} M_{\alpha}\right)$, albite (Na $\left.K_{\alpha}\right)$, orthoclase $\left(\mathrm{K} K_{\alpha}\right), \operatorname{LiF}\left(\mathrm{F} K_{\alpha}\right)$, and $\mathrm{NaCl}\left(\mathrm{Cl} K_{\alpha}\right)$. Special care was taken to ensure that line overlaps were properly corrected and that background positions were clear of interferences among the REE. We used empirically determined correction factors applied to the following line overlaps: Th $\rightarrow \mathrm{U}$, Dy $\rightarrow \mathrm{Eu}$, $\mathrm{Gd} \rightarrow \mathrm{Ho}, \mathrm{La} \rightarrow \mathrm{Gd}, \mathrm{Ce} \rightarrow$ $\mathrm{Gd}, \mathrm{Eu} \rightarrow \mathrm{Er}, \mathrm{Gd} \rightarrow \mathrm{Er}, \mathrm{Sm} \rightarrow$ $\mathrm{Tm}, \mathrm{Dy} \rightarrow \mathrm{Lu}, \mathrm{Ho} \rightarrow \mathrm{Lu}, \mathrm{Yb}$ $\rightarrow \mathrm{Lu}$, and Dy $\rightarrow$ As (Konečný et al. 2004). Analyses used for uraninite dating were carried out at different conditions to maximize the accuracy of $\mathrm{Pb}$ measurement and hence of the age calculation. An accelerating voltage of $15 \mathrm{kV}$ and focused beam of $3 \mu \mathrm{m}$ diameter were used. The $\mathrm{Pb}$ was measured at $\mathrm{Pb} \mathrm{M}_{\alpha}$ line. Counting time increased to $300 \mathrm{~s}$ (peak and background) for $\mathrm{Pb}, 80 \mathrm{~s}$ for $\mathrm{U}$ and $35 \mathrm{~s}$ for Th and high sample current of $180 \mathrm{nA}$ were considered reasonable compromise conditions between the degree of surface damage and analytical accuracy. Other elements and lines involved in the age calculation were $\operatorname{Th} M_{\alpha}$ and $\mathrm{U} M_{\beta}$. Interferences $\mathrm{U} M_{\beta}^{\alpha}$ with (Th $M_{\alpha}$, Th $M 3-N 4$, Th M5-P3) and $\mathrm{Pb} M_{\alpha}$ with (Th $M_{\zeta 1}$ Th $\left.M_{\zeta 2}\right)$ and $\mathrm{Pb} M_{\alpha}\left(\mathrm{Y}_{\gamma 2,3}\right)$ were accounted for by empirically derived coefficients (Amli and Griffin 1975). The matrix effects were corrected by the PAP procedure.

Florencite and goyazite formulae were calculated on the basis of six cations in the formula unit $\left[\left(\mathrm{M}^{2+}, \mathrm{REE}\right)+(\mathrm{Al}, \mathrm{Fe})\right.$ $+\mathrm{P}=6]$, following the proce-
Tab. 1 Chemical composition of fluorapatite from Čučma (wt. \% and $a p f u$ )

\begin{tabular}{|c|c|c|c|c|c|c|c|c|}
\hline & 1 & 2 & 3 & 4 & 5 & 6 & 7 & 8 \\
\hline $\mathrm{P}_{2} \mathrm{O}_{5}$ & 41.43 & 41.39 & 41.32 & 39.83 & 41.00 & 40.70 & 40.85 & 39.95 \\
\hline $\mathrm{SiO}_{2}$ & 0.12 & 0.15 & 0.05 & 0.18 & 0.34 & 0.23 & 0.10 & 0.39 \\
\hline $\mathrm{ThO}_{2}$ & 0.03 & 0.00 & 0.13 & 0.50 & 0.20 & 0.03 & 0.00 & 0.00 \\
\hline $\mathrm{Y}_{2} \mathrm{O}_{3}$ & 0.19 & 0.31 & 0.00 & 0.55 & 0.08 & 0.44 & 0.23 & 0.10 \\
\hline $\mathrm{La}_{2} \mathrm{O}_{3}$ & 0.04 & 0.09 & 0.04 & 0.31 & 0.06 & 0.07 & 0.03 & 0.06 \\
\hline $\mathrm{Ce}_{2} \mathrm{O}_{3}$ & 0.19 & 0.20 & 0.14 & 0.71 & 0.23 & 0.27 & 0.11 & 0.18 \\
\hline $\mathrm{Pr}_{2} \mathrm{O}_{3}$ & 0.09 & 0.00 & 0.00 & 0.23 & 0.08 & 0.08 & 0.06 & 0.04 \\
\hline $\mathrm{Nd}_{2} \mathrm{O}_{3}$ & 0.07 & 0.23 & 0.06 & 0.43 & 0.28 & 0.05 & 0.27 & 0.15 \\
\hline $\mathrm{Sm}_{2} \mathrm{O}_{3}$ & 0.00 & 0.09 & 0.00 & 0.21 & 0.04 & 0.08 & 0.09 & 0.07 \\
\hline $\mathrm{Eu}_{2} \mathrm{O}_{3}$ & 0.21 & 0.11 & 0.06 & 0.22 & 0.07 & 0.13 & 0.00 & 0.13 \\
\hline $\mathrm{FeO}$ & 0.09 & 0.24 & 0.04 & 0.02 & 0.29 & 0.13 & 0.39 & 1.09 \\
\hline $\mathrm{MnO}$ & 0.04 & 0.53 & 0.06 & 0.01 & 0.03 & 1.14 & 0.87 & 0.02 \\
\hline $\mathrm{MgO}$ & 0.01 & 0.03 & 0.04 & 0.00 & 0.02 & 0.00 & 0.02 & 0.00 \\
\hline $\mathrm{CaO}$ & 56.04 & 55.29 & 56.54 & 54.79 & 55.97 & 53.76 & 54.36 & 54.72 \\
\hline $\mathrm{SrO}$ & 0.02 & 0.00 & 0.03 & 0.04 & 0.00 & 0.00 & 0.00 & 0.00 \\
\hline $\mathrm{H}_{2} \mathrm{O}$ calc. & 0.01 & 0.09 & 0.20 & 0.44 & 0.22 & 0.06 & 0.09 & 0.08 \\
\hline $\mathrm{F}$ & 3.76 & 3.58 & 3.36 & 2.78 & 3.30 & 3.55 & 3.52 & 3.55 \\
\hline Total & 102.33 & 102.25 & 101.89 & 100.80 & 101.98 & 100.67 & 100.90 & 100.48 \\
\hline $\mathrm{O}=\mathrm{F}$ & -1.88 & -1.79 & -1.68 & -1.39 & -1.65 & -1.78 & -1.76 & -1.78 \\
\hline Total & 100.45 & 100.45 & 100.21 & 99.40 & 100.32 & 98.86 & 99.11 & 98.70 \\
\hline$\overline{\mathrm{P}^{5+}}$ & 2.932 & 2.934 & 2.918 & 2.875 & 2.903 & 2.937 & 2.932 & 2.877 \\
\hline $\mathrm{Si}^{4+}$ & 0.010 & 0.012 & 0.004 & 0.015 & 0.028 & 0.020 & 0.009 & 0.033 \\
\hline Sum $T$ & 2.942 & 2.947 & 2.922 & 2.890 & 2.931 & 2.957 & 2.941 & 2.910 \\
\hline $\mathrm{Th}^{4+}$ & 0.001 & 0.000 & 0.003 & 0.010 & 0.004 & 0.001 & 0.000 & 0.000 \\
\hline $\mathrm{Y}^{3+}$ & 0.009 & 0.014 & 0.000 & 0.025 & 0.004 & 0.020 & 0.010 & 0.005 \\
\hline $\mathrm{La}^{3+}$ & 0.001 & 0.003 & 0.001 & 0.010 & 0.002 & 0.002 & 0.001 & 0.002 \\
\hline $\mathrm{Ce}^{3+}$ & 0.006 & 0.006 & 0.004 & 0.022 & 0.007 & 0.009 & 0.003 & 0.006 \\
\hline $\operatorname{Pr}^{3+}$ & 0.003 & 0,000 & 0.000 & 0.007 & 0.002 & 0.003 & 0.002 & 0.001 \\
\hline $\mathrm{Nd}^{3+}$ & 0.002 & 0.007 & 0.002 & 0.013 & 0.008 & 0.001 & 0.008 & 0.005 \\
\hline $\mathrm{Sm}^{3+}$ & 0.000 & 0.003 & 0.001 & 0.006 & 0.001 & 0.002 & 0.003 & 0.002 \\
\hline $\mathrm{Eu}^{3+}$ & 0.006 & 0.003 & 0.002 & 0.006 & 0.002 & 0.004 & 0.000 & 0.004 \\
\hline $\mathrm{Fe}^{2+}$ & 0.006 & 0.017 & 0.003 & 0.001 & 0.020 & 0.009 & 0.028 & 0.077 \\
\hline $\mathrm{Mn}^{2+}$ & 0.003 & 0.037 & 0.004 & 0.001 & 0.002 & 0.082 & 0.062 & 0.002 \\
\hline $\mathrm{Mg}^{2+}$ & 0.001 & 0.003 & 0.005 & 0.000 & 0.002 & 0.000 & 0.003 & 0.000 \\
\hline $\mathrm{Ca}^{2+}$ & 5.020 & 4.960 & 5.053 & 5.006 & 5.015 & 4.910 & 4.939 & 4.978 \\
\hline $\mathrm{Sr}^{2+}$ & 0.001 & 0.000 & 0.001 & 0.002 & 0,000 & 0.000 & 0.000 & 0.000 \\
\hline Sum $M$ & 5.058 & 5.053 & 5.078 & 5.110 & 5.069 & 5.043 & 5.059 & 5.090 \\
\hline $\mathrm{OH}^{-}$ & 0.006 & 0.050 & 0.113 & 0.249 & 0.124 & 0.035 & 0.048 & 0.043 \\
\hline $\mathrm{F}^{-}$ & 0.994 & 0.950 & 0.886 & 0.751 & 0.876 & 0.965 & 0.952 & 0.957 \\
\hline $\operatorname{Sum} X$ & 1.000 & 1.000 & 1.000 & 1.000 & 1.000 & 1.000 & 1.000 & 1.000 \\
\hline
\end{tabular}

Formulae based on 12 oxygen atoms and $\mathrm{OH}+\mathrm{F}=1$ apfu

Contents of As, Ti, U, Al, Sc, $\mathrm{Pb}, \mathrm{Cl}$ are below the detection limit dure applied to florencite by Janeczek and Ewing (1996). The total number of ions of $\mathrm{OH}^{-}$replacing $\mathrm{O}^{2-}$ was estimated by charge balance from $\mathrm{OH}=22-(\Sigma$ cation charges), and the amount of $\mathrm{O}^{2-}$ replaced by $\mathrm{OH}^{-}$in $\mathrm{PO}_{4}$ tetrahedra from $\mathrm{O}=4-\mathrm{OH}$. The weight concentration of $\mathrm{H}_{2} \mathrm{O}$ was then calculated from the amount of $\mathrm{OH}$ obtained.

Monazite and xenotime formulae were obtained on the basis of 4 oxygen atoms. Apatite was recast to give 13 total anions assuming $\mathrm{OH}=1-(\mathrm{F}+\mathrm{Cl})$ and uraninite formulae were calculated based on sum of cations $=1$ atom. 

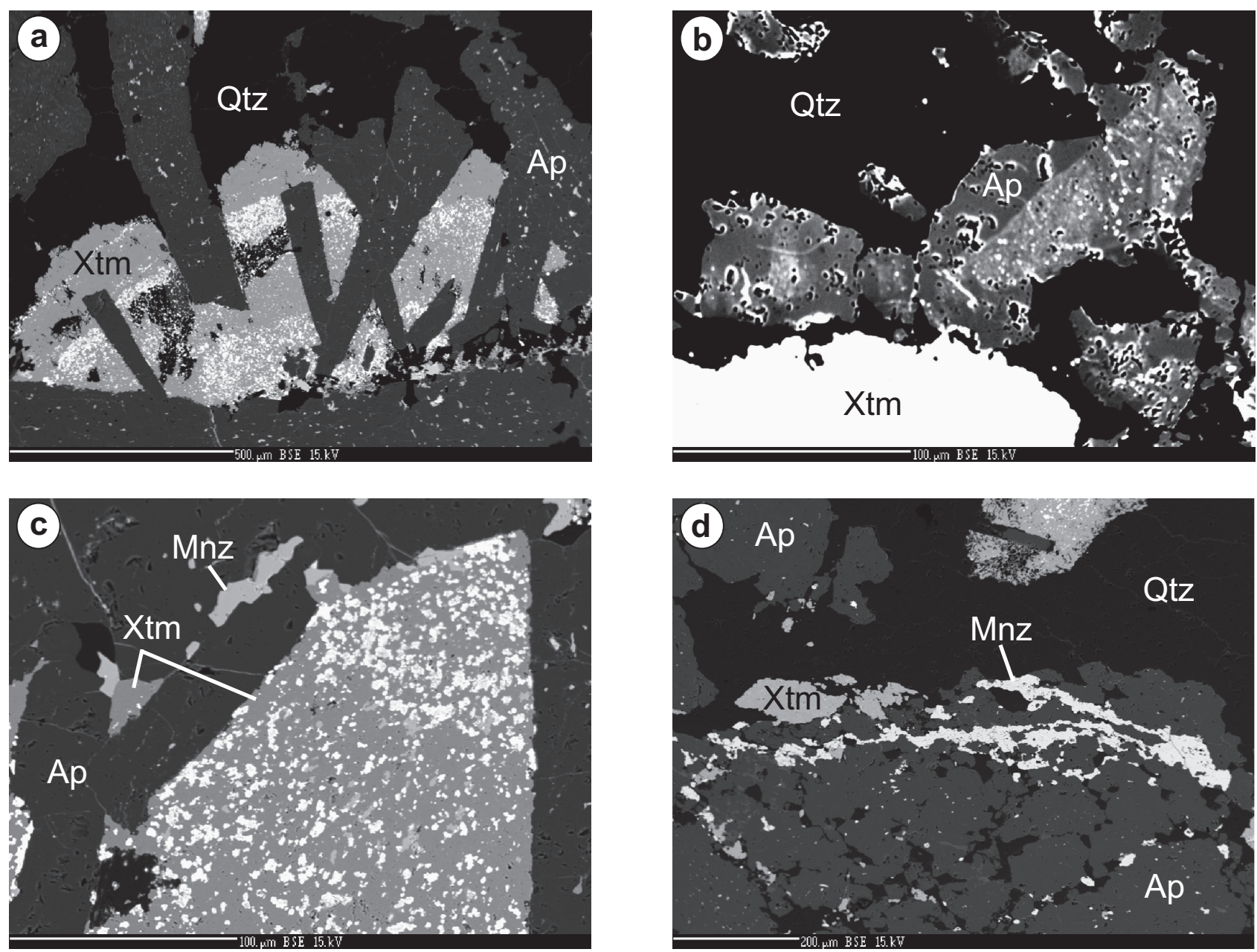

Fig. 2a - Prismatic to columnar crystals of fluorapatite (Ap) associated with xenotime-(Y) (Xtm) and uraninite (white) in quartz (Qtz), BEI. b - Fluorapatite crystals (Ap) with sector zoning in association with xenotime-(Y) (Xtm) and quartz (Qtz), BEI. c - Subhedral to anhedral crystals of monazite-(Ce) to monazite-(Nd) (Mnz) associated with two different types of xenotime-(Y) (Xtm), uraninite (white), fluorapatite (Ap) and minor quartz (black), BEI. d - Veinlet of monazite-(Ce) (Mnz) in fluorapatite (Ap) with xenotime-(Y) (Xtm), uraninite (white) and quartz (Qtz), BEI.

\section{Results}

\subsection{Quartz}

Massive grey quartz is the dominant mineral of the REE-U mineralization. It forms anhedral monomineral aggregates, hosting fluorapatite, REE phosphates, and other minerals. Locally, quartz contains minute veinlets and aggregates of muscovite, Fe-oxide phase (hematite or magnetite), and uraninite. Using cold cathodoluminescence (CL) imaging, quartz shows thin irregular aureole with brighter CL around the microscopic uraninite veinlets, apparently due to local radiation damage.

\subsection{Fluorapatite}

Fluorapatite is very common and the most characteristic mineral at the studied locality. It forms prismatic to columnar crystals up to $3 \mathrm{~cm}$ long, which are usually concentrated to parallel bands in quartz (Fig. 2a). Rare partially corroded crystals of fluorapatite up to $5 \mathrm{~mm}$ across were observed in cavities of quartz. Locally, it shows irregular to sector zoning (Fig. 2b). Fractures and interstitial space between fluorapatite crystals are filled by younger crystals and aggregates of monazite- $(\mathrm{Ce})$ and monazite-(Nd), xenotime-(Y), uraninite, coffinite, and supergene minerals, like florencite-(Ce), goyazite, and autunite. Tiny inclusions of above-mentioned minerals in fluorapatite are also common. Fluorapatite shows high $\mathrm{F}$ (0.72 to $1.00 \mathrm{apfu}$ ) and locally elevated $\mathrm{Fe}$ and Mn contents (up to 1.1 wt. \% $\mathrm{FeO}$ and $\mathrm{MnO} ; 0.08$ apfu $\mathrm{Fe}$ and $\mathrm{Mn})$. These contents are generally lower than $\mathrm{Fe}$ and $\mathrm{Mn}$ concentrations in accessory magmatic fluorapatite I ( $\leq$ 1.4 wt. $\% \mathrm{FeO}$ and $1.6-3.3$ wt. \% $\mathrm{MnO}$ ) but higher than secondary low-T fluorapatite II, both from adjacent granites $(<0.1$ wt. $\% \mathrm{FeO}$ and $<0.5$ wt. \% $\mathrm{MnO}$; Broska et al. 2004). Consequently, the concentrations of $\mathrm{Fe}$ and $\mathrm{Mn}$ in 
Quartz-apatite-REE phosphates-uraninite vein mineralization near Čučma, Slovakia

fluorapatite generally decreased from evolved magmatic stage to post-magmatic (hydrothermal) high- to low-T conditions in the studied area. On the other hand, concentrations of $\mathrm{Y}, \mathrm{REE}, \mathrm{Si}$, Th, U, and Sr are generally low: up to 1 wt. $\% \mathrm{Y}_{2} \mathrm{O}_{3}(0.05$ apfu $)$, $\leq 0.7$ wt. $\% \mathrm{Ce}_{2} \mathrm{O}_{3}(0.02$ apfu $)$, $\leq 0.3$ wt. $\% \mathrm{La}_{2} \mathrm{O}_{3}$ (0.01 apfu), $\leq 0.2$ wt. $\%$ of other REE, $\leq 0.5$ wt. $\% \mathrm{ThO}_{2}, \leq 0.3$ wt. $\% \mathrm{UO}_{2}$, and $\leq 0.1$ wt. $\%$ SrO (Tab. 1 ).

\subsection{Monazite-(Ce) to monazite- $(\mathrm{Nd})$}

Monazite- $(\mathrm{Ce})$ to monazite$(\mathrm{Nd})$ form aggregates of isolated subhedral to anhedral crystals up to $80 \mu \mathrm{m}$ (Fig. 2c) or up to $600 \mu \mathrm{m}$ long veinlets (Fig. 2d) in botryoidal aggregates of xenotime-(Y) or fluorapatite and quartz. Both monazite-(Ce) and monazite-( $\mathrm{Nd})$ are often associated with crystals of xenotime-(Y), coffinite, uraninite, and secondary minerals, e.g., florencite-(Ce), goyazite, and autunite. Slight irregular zoning of monazite is caused mainly by variable $\mathrm{Ce}$ and $\mathrm{Nd}$ contents.

Monazite has a relatively homogenous composition dominated by the LREE phosphate end-member and contains only subordinate amounts of the cheralite $\left(\mathrm{Chr}, \mathrm{Ca}_{0.5} \mathrm{Th}_{0.5} \mathrm{PO}_{4}\right)$ and huttonite (Hut, $\mathrm{ThSiO}_{4}$ ) components $\left(\mathrm{X}_{\mathrm{Chr}} \leq 10 \mathrm{~mol} . \%\right.$, $\mathrm{X}_{\text {Hut }}=0$ mol. \%; Tab. 2). The $\mathrm{Ce}^{3+}$ is the dominant REE cation (0.30-0.50 apfu Ce). However monazite-(Nd) with atomic $\mathrm{Nd} /$ Ce of 1.02-1.23 and 0.35-0.38 apfu $\mathrm{Nd}$ was detected in places (Fig. 3; Tab. 2). Locally, monazite-(Nd) shows slightly elevated content of $\mathrm{S}$ (up to 0.9 wt. $\% \mathrm{SO}_{3} ; 0.02$ apfu $\mathrm{S}$ ). The content of Th in monazite is usually below the detection limit of the electron microprobe
Tab. 2 Representative electron-microprobe analyses of monazite-(Ce), monazite-(Nd), and xenotime-(Y) (wt. $\%$ and $a p f u$ )

\begin{tabular}{|c|c|c|c|c|c|c|c|c|}
\hline Mineral & Mnz-Ce & $\mathrm{Mnz}-\mathrm{Ce}$ & Mnz-Nd & Mnz-Nd & Xtm & Xtm & Xtm & Xtm \\
\hline $\mathrm{SO}_{3}$ & 0.03 & 0.07 & 0.92 & 0.05 & 0.02 & 0.015 & 0.03 & 0.01 \\
\hline $\mathrm{P}_{2} \mathrm{O}_{5}^{3}$ & 29.43 & 30.51 & 28.83 & 30.13 & 34.64 & 34.09 & 34.61 & 34.35 \\
\hline $\mathrm{As}_{2}^{2} \mathrm{O}_{5}$ & 0.22 & 0.21 & 0.23 & 0.27 & 0.02 & 0.01 & 0.00 & 0.01 \\
\hline $\mathrm{SiO}_{2}{ }_{2}^{5}$ & 0.15 & 0.15 & 0.22 & 0.19 & 0.29 & 0.22 & 0.12 & 0.11 \\
\hline $\mathrm{ThO}_{2}^{2}$ & 0.00 & 0.03 & 0.00 & 0.00 & 0.03 & 0.02 & 0.05 & 0.01 \\
\hline $\mathrm{UO}_{2}{ }_{2}^{2}$ & 0.04 & 0.60 & 0.23 & 0.15 & 0.60 & 0.64 & 0.39 & 0.37 \\
\hline $\mathrm{TiO}_{2}^{2}$ & 0.00 & 0.03 & 0.00 & 0.01 & n.a. & n.a. & n.a. & n.a. \\
\hline $\mathrm{Sc}_{2} \mathrm{O}_{3}$ & 0.00 & 0.00 & 0.00 & 0.01 & n.a. & n.a. & n.a. & n.a. \\
\hline $\mathrm{Al}_{2}^{2} \mathrm{O}_{3}^{3}$ & 0.00 & 0.00 & 0.01 & 0.02 & 0.00 & 0.00 & 0.00 & 0.00 \\
\hline $\mathrm{Y}_{2} \mathrm{O}_{3}{ }^{3}$ & 0.27 & 0.69 & 0.73 & 1.16 & 39.07 & 39.29 & 40.02 & 40.29 \\
\hline $\mathrm{La}_{2}^{2} \mathrm{O}_{3}^{3}$ & 9.12 & 8.55 & 7.28 & 6.76 & 0.00 & 0.00 & 0.00 & 0.00 \\
\hline $\mathrm{Ce}_{2} \mathrm{O}_{3}$ & 26.19 & 26.69 & 22.44 & 20.75 & 0.05 & 0.03 & 0.09 & 0.07 \\
\hline $\mathrm{Pr}_{2} \mathrm{O}_{3}$ & 4.08 & 4.01 & 4.27 & 4.20 & 0.17 & 0.10 & 0.12 & 0.12 \\
\hline $\mathrm{Nd}_{2} \mathrm{O}_{3}$ & 20.96 & 19.46 & 24.25 & 25.14 & 0.40 & 0.31 & 0.18 & 0.17 \\
\hline $\mathrm{Sm}_{2}^{2} \mathrm{O}_{3}^{3}$ & 5.96 & 4.80 & 5.82 & 6.50 & 1.17 & 1.19 & 1.15 & 1.10 \\
\hline $\mathrm{Eu}_{2} \mathrm{O}_{3}^{3}$ & 0.32 & 0.60 & 0.27 & 0.40 & 0.22 & 0.35 & 0.40 & 0.44 \\
\hline $\mathrm{Gd}_{2}^{2} \mathrm{O}_{3}^{3}$ & 1.10 & 2.16 & 2.33 & 2.74 & 3.91 & 4.29 & 4.41 & 4.19 \\
\hline $\mathrm{Tb}_{2}^{2} \mathrm{O}_{3}^{3}$ & 0.05 & 0.19 & 0.09 & 0.09 & 0.83 & 0.94 & 0.93 & 0.87 \\
\hline $\mathrm{Dy}_{2} \mathrm{O}_{3}$ & 0.23 & 0.43 & 0.50 & 0.64 & 6.33 & 6.58 & 6.58 & 6.49 \\
\hline $\mathrm{Ho}_{2} \mathrm{O}_{3}$ & 0.00 & 0.000 & 0.06 & 0.09 & 1.04 & 1.07 & 1.03 & 1.07 \\
\hline $\mathrm{Er}_{2} \mathrm{O}_{3}{ }_{3}$ & 0.32 & 0.272 & 0.29 & 0.37 & 3.79 & 3.76 & 3.82 & 3.80 \\
\hline $\mathrm{Tm}_{2}^{2} \mathrm{O}_{3}$ & 0.08 & 0.20 & 0.10 & 0.26 & 0.63 & 0.58 & 0.63 & 0.62 \\
\hline $\mathrm{Yb}_{2} \mathrm{O}_{3}^{3}$ & 0.08 & 0.13 & 0.16 & 0.15 & 2.94 & 2.96 & 3.04 & 3.16 \\
\hline $\mathrm{Lu}_{2} \mathrm{O}_{3}$ & 0.01 & 0.25 & 0.09 & 0.08 & 0.40 & 0.40 & 0.40 & 0.40 \\
\hline $\mathrm{CaO}^{3}$ & 0.46 & 0.42 & 0.24 & 0.27 & 0.06 & 0.08 & 0.04 & 0.02 \\
\hline $\mathrm{SrO}$ & 0.03 & 0.00 & 0.03 & 0.03 & 0.01 & 0.02 & 0.00 & 0.03 \\
\hline $\mathrm{BaO}$ & 0.00 & 0.07 & 0.00 & 0.06 & n.a. & n.a. & n.a. & n.a. \\
\hline $\mathrm{MnO}$ & 0.09 & 0.07 & 0.08 & 0.07 & 0.00 & 0.00 & 0.00 & 0.00 \\
\hline $\mathrm{FeO}$ & 0.00 & 0.01 & 0.00 & 0.00 & 0.01 & 0.04 & 0.00 & 0.02 \\
\hline $\mathrm{PbO}$ & 0.00 & 0.03 & 0.00 & 0.00 & 0.00 & 0.00 & 0.00 & 0.00 \\
\hline $\mathrm{Na}_{2} \mathrm{O}$ & n.a. & n.a. & n.a. & n.a. & 0.00 & 0.00 & 0.00 & 0.00 \\
\hline $\mathrm{K}_{2} \mathrm{O}$ & n.a. & n.a. & n.a. & n.a. & 0.00 & 0.00 & 0.00 & 0.00 \\
\hline $\mathrm{F}^{2}$ & 0.00 & 0.00 & 0.00 & 0.00 & 0.00 & 0.00 & 0.00 & 0.00 \\
\hline $\mathrm{Cl}$ & 0.05 & 0.05 & 0.08 & 0.06 & 0.02 & 0.02 & 0.01 & 0.01 \\
\hline Total & 100.17 & 100.66 & 99.55 & 100.62 & 96.64 & 96.99 & 98.07 & 97.72 \\
\hline $\mathrm{O}=\mathrm{F}$ & 0.00 & 0.00 & 0.00 & 0.00 & 0.00 & 0.00 & 0.00 & 0.00 \\
\hline $\mathrm{O}=\mathrm{Cl}$ & -0.01 & -0.01 & -0.02 & -0.01 & -0.01 & -0.01 & -0.00 & -0.00 \\
\hline Total & 100.16 & 100.65 & 99.53 & 100.61 & 96.64 & 96.99 & 98.06 & 97.72 \\
\hline $\mathrm{S}^{6+}$ & 0.001 & 0.002 & 0.028 & 0.002 & 0.000 & 0.000 & 0.001 & 0.000 \\
\hline $\mathrm{P}^{5+}$ & 1.005 & 1.021 & 0.982 & 1.015 & 1.030 & 1.020 & 1.024 & 1.021 \\
\hline $\mathrm{As}^{5+}$ & 0.005 & 0.004 & 0.005 & 0.006 & 0.000 & 0.000 & 0.000 & 0.000 \\
\hline $\mathrm{Si}^{4+}$ & 0.006 & 0.006 & 0.009 & 0.008 & 0.010 & 0.008 & 0.004 & 0.004 \\
\hline $\mathrm{Th}^{4+}$ & 0.000 & 0.000 & 0.000 & 0.000 & 0.000 & 0.000 & 0.000 & 0.000 \\
\hline $\mathrm{U}^{4+}$ & 0.000 & 0.005 & 0.002 & 0.001 & 0.005 & 0.005 & 0.003 & 0.003 \\
\hline $\mathrm{Ti}^{4+}$ & 0.000 & 0.001 & 0,000 & 0.000 & n.a. & n.a. & n.a. & n.a. \\
\hline $\mathrm{Sc}^{3+}$ & 0.000 & 0.000 & 0.000 & 0.000 & n.a. & n.a. & n.a. & n.a. \\
\hline $\mathrm{Al}^{3+}$ & 0.000 & 0.000 & 0.000 & 0.000 & 0.000 & 0.000 & 0.000 & 0.000 \\
\hline $\mathrm{Y}^{3+}$ & 0.006 & 0.014 & 0.016 & 0.025 & 0.730 & 0.739 & 0.744 & 0.753 \\
\hline $\mathrm{La}^{3+}$ & 0.136 & 0.125 & 0.108 & 0.099 & 0.000 & 0.000 & 0.000 & 0.000 \\
\hline $\mathrm{Ce}^{3+}$ & 0.387 & 0.386 & 0.330 & 0.302 & 0.001 & 0.000 & 0.001 & 0.001 \\
\hline $\operatorname{Pr}^{3+}$ & 0.060 & 0.058 & 0.063 & 0.061 & 0.002 & 0.001 & 0.001 & 0.002 \\
\hline $\mathrm{Nd}^{3+}$ & 0.302 & 0.275 & 0.348 & 0.357 & 0.005 & 0.004 & 0.002 & 0.002 \\
\hline $\mathrm{Sm}^{3+}$ & 0.083 & 0.065 & 0.081 & 0.089 & 0.014 & 0.015 & 0.014 & 0.013 \\
\hline $\mathrm{Eu}^{3+}$ & 0.004 & 0.008 & 0.004 & 0.005 & 0.003 & 0.004 & 0.005 & 0.005 \\
\hline $\mathrm{Gd}^{3+}$ & 0.027 & 0.028 & 0.031 & 0.036 & 0.046 & 0.050 & 0.051 & 0.049 \\
\hline $\mathrm{Tb}^{3+}$ & 0.001 & 0.002 & 0.001 & 0.001 & 0.010 & 0.011 & 0.011 & 0.010 \\
\hline $\mathrm{Dy}^{3+}$ & 0.003 & 0.005 & 0.007 & 0.008 & 0.072 & 0.075 & 0.074 & 0.073 \\
\hline $\mathrm{Ho}^{3+}$ & 0.000 & 0.000 & 0.001 & 0.001 & 0.012 & 0.012 & 0.011 & 0.012 \\
\hline $\mathrm{Er}^{3+}$ & 0.004 & 0.003 & 0.004 & 0.005 & 0.042 & 0.042 & 0.042 & 0.042 \\
\hline $\mathrm{Tm}^{3+}$ & 0.001 & 0.002 & 0.001 & 0.003 & 0.007 & 0.006 & 0.007 & 0.007 \\
\hline $\mathrm{Yb}^{3+}$ & 0.001 & 0.002 & 0.002 & 0.002 & 0.031 & 0.032 & 0.032 & 0.034 \\
\hline $\mathrm{Lu}^{3+}$ & 0.000 & 0.003 & 0.001 & 0.001 & 0.004 & 0.004 & 0.004 & 0.004 \\
\hline $\mathrm{Ca}^{2+}$ & 0.020 & 0.018 & 0.011 & 0.012 & 0.002 & 0.003 & 0.001 & 0.001 \\
\hline $\mathrm{Sr}^{2+}$ & 0.001 & 0.000 & 0.001 & 0.001 & 0.000 & 0.000 & 0.000 & 0.001 \\
\hline $\mathrm{Ba}^{2+}$ & 0.000 & 0.001 & 0.000 & 0.001 & 0.000 & 0.000 & 0.000 & 0.000 \\
\hline $\mathrm{Mn}^{2+}$ & 0.003 & 0.002 & 0.003 & 0.002 & 0.000 & 0.000 & 0.000 & 0.000 \\
\hline $\mathrm{Fe}^{2+}$ & 0.000 & 0.000 & 0.000 & 0.000 & 0.000 & 0.001 & 0.000 & 0.000 \\
\hline $\mathrm{Pb}^{2+}$ & 0.000 & 0.000 & 0.000 & 0.000 & 0.000 & 0.000 & 0.000 & 0.000 \\
\hline $\mathrm{Na}^{+}$ & n.a. & n.a. & n.a. & n.a. & 0.000 & 0.000 & 0.000 & 0.000 \\
\hline $\mathrm{K}^{+}$ & n.a. & n.a. & n.a. & n.a. & 0.000 & 0.000 & 0.000 & 0.000 \\
\hline Total & 2.055 & 2.040 & 2.037 & 2.043 & 2.026 & 2.035 & 2.034 & 2.036 \\
\hline position A & 1.038 & 1.006 & 1.013 & 1.014 & 0.985 & 1.006 & 1.005 & 1.011 \\
\hline position B & 1.017 & 1.033 & 1.023 & 1.029 & 1.041 & 1.029 & 1.028 & 1.025 \\
\hline$X$ (Monazite) & 0.961 & 0.964 & 0.979 & 0.977 & & & & \\
\hline$X$ (Cheralite) & 0.039 & 0.036 & 0.021 & 0.023 & & & & \\
\hline$X$ (Huttonite) & 0.000 & 0.000 & 0.000 & 0.000 & & & & \\
\hline
\end{tabular}




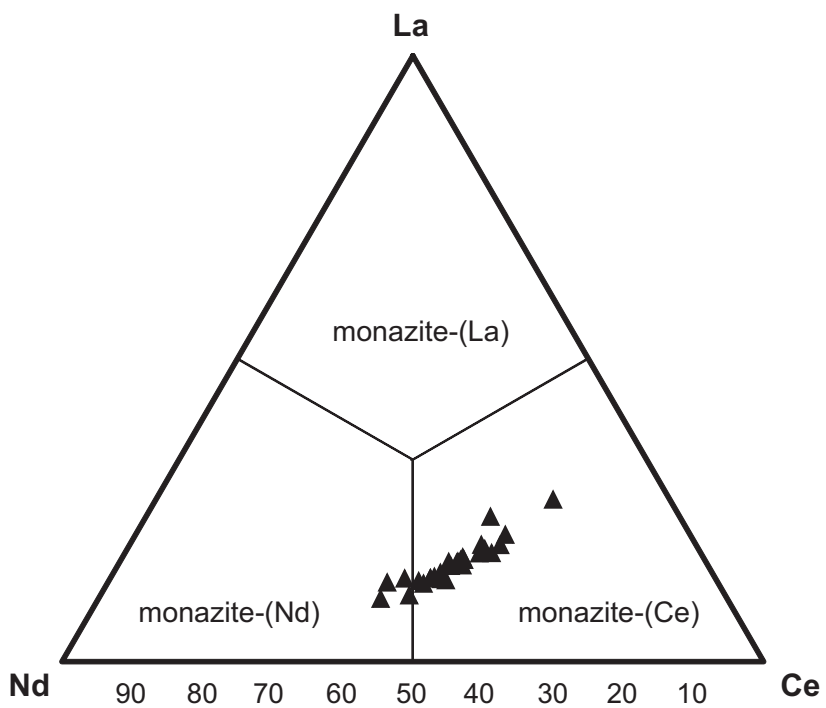

Fig. 3 Composition of monazite from Čučma in the Nd-La-Ce ternary diagram (atomic proportions).

(up to 0.02 wt. $\% \mathrm{ThO}_{2}$ ); also concentration of $\mathrm{U}$ (up to 0.7 wt. $\% \mathrm{UO}_{2} ; 0.007$ apfu $\left.\mathrm{U}\right)$ and other measured elements (As, $\mathrm{Si}, \mathrm{Fe}, \mathrm{Ca}, \mathrm{Sr}, \mathrm{Al}$ and $\mathrm{Ti}$ ) are negligible to low
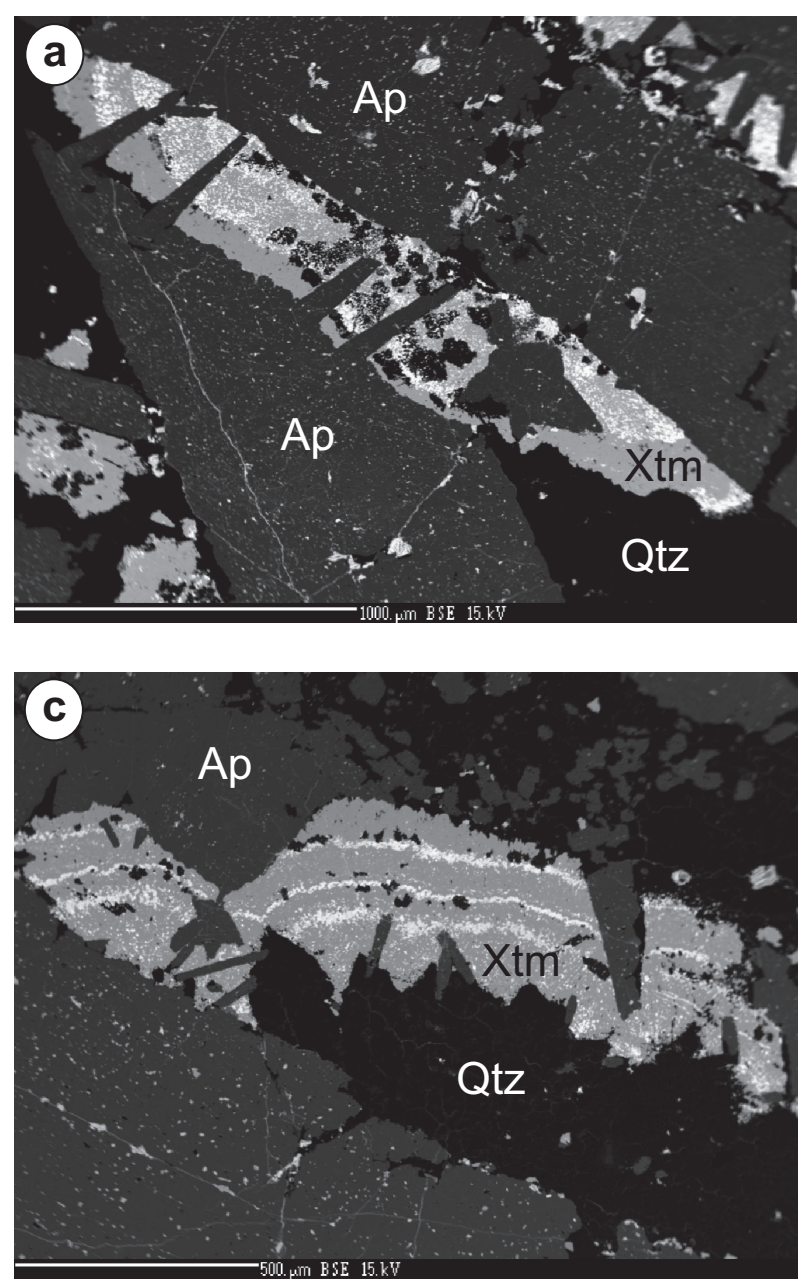

(Tab. 2). Consequently, our attempt to apply chemical, in situ electron-microprobe dating of monazite was unsuccessful due to very low Th and U contents.

\subsection{Xenotime- $(Y)$}

Xenotime-(Y) represents the most abundant primary REE phosphate at the studied locality. Two types of xenotime(Y) were observed, which differ in form as well as in chemical composition. The first and the most common type is represented by colloform to botryoidal aggregates (Fig. 4a) up to $2 \mathrm{~mm}$ in size, which fill the interstitial space between the older fluorapatite crystals. Typical of these aggregates are numerous tiny inclusions of uraninite and monazite-(Ce), which are mostly parallel to the banding of xenotime-(Y) aggregates (Fig. 4a). The second type of xenotime-(Y) are subhedral crystals up to $90 \mu \mathrm{m}$ across, which are grouped to irregular aggregates in quartz or in fluorapatite (Fig. 4b). This type of xenotime-(Y) is commonly associated with monazite-(Ce), monazite-(Nd), and uraninite. The first population of xenotime-(Y) shows a relatively uniform chemical composition with 39 to 43 wt. $\% \mathrm{Y}_{2} \mathrm{O}_{3}(0.73$ to $0.77 a p f u \mathrm{Y})$, atomic ratio $\mathrm{Y} /\left(\sum\right.$

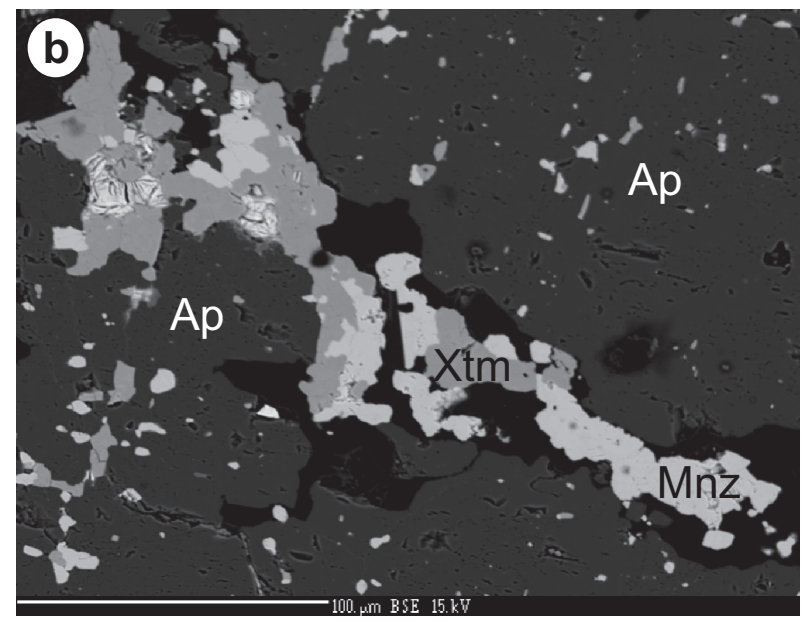

Fig. 4a - Botryoidal aggregate of xenotime-(Y) (Xtm) with numerous uraninite inclusions (white) in association with fluorapatite (dark grey) and quartz (Qtz), BEI. b - Subhedral crystals of xenotime-(Y) (Xtm) associated with monazite-(Ce) and monazite-(Nd) (Mnz) in fracture of fluorapatite (Ap), BEI. c - Parallel bands of uraninite inclusions (white) in botryoidal aggregates of xenotime-(Y) $(\mathrm{Xtm})$ associated with fluorapatite (Ap) and quartz (Qtz), BEI. 
Quartz-apatite-REE phosphates-uraninite vein mineralization near Čučma, Slovakia

$\mathrm{Y}+\mathrm{REE})=0.76-0.79$, and small to negligible contents of Th, $\mathrm{U}$, $\mathrm{Zr}, \mathrm{Hf}, \mathrm{Ca}$, and radiogenic $\mathrm{Pb}$ (Tab. 2). The second type of xenotime-(Y) differs in lower concentration of Y (up to $29 \mathrm{wt}$. $\% \mathrm{Y}_{2} \mathrm{O}_{3} ; 0.63$ apfu $\mathrm{Y}$ ) compensated by an enrichment of LREE and MREE, mainly $\mathrm{Nd}, \mathrm{Sm}$, and Gd (up to 5 wt. $\% \mathrm{Nd}_{2} \mathrm{O}_{3} ; 0.07$ apfu $\mathrm{Nd}$, up to 6.9 wt. $\% \mathrm{Sm}_{2} \mathrm{O}_{3}$; 0.1 apfu $\mathrm{Sm}$ and up to $7.2 \mathrm{wt}$. $\% \mathrm{Gd}_{2} \mathrm{O}_{3} ; 0.1$ apfu $\mathrm{Gd}$, respectively). All the analyses gave low totals ( $\sim 90$ to 93 wt. \%, Tab. 2). Thus, these compositions probably represent a partly hydrated xenotime and/or possibly hydrated yttrium phosphate (churchite, $\mathrm{YPO}_{4} \cdot 2 \mathrm{H}_{2} \mathrm{O}$, or rhabdophane-(Y), $\left.\mathrm{YPO}_{4} \cdot \mathrm{H}_{2} \mathrm{O}\right)$ in which elevated MREE contents are a typical feature (Plášil et al. 2009).

\subsection{Uraninite}

Uraninite is an abundant mineral and it forms anhedral crystals and aggregates, typically up to $50 \mu \mathrm{m}$ in size, exceptionally up to $2 \mathrm{~mm}$ which are associated mostly with xenotime-(Y), and monazite-(Ce) to monazite$(\mathrm{Nd})$. Characteristic are parallel bands of uraninite inclusions (Fig. 4c) in botryoidal aggregates of xenotime-(Y). Uraninite is often altered to supergene minerals, especially autunite to meta-autunite. It shows a relatively uniform chemical composition that is close to the theoretical end member. Uranium contents attain 0.87 to 0.91 apfu, whereas Th concentrations are very low, under detection limit of the electron microprobe (Tab. 3). Lead concentrations vary between 2.0 and 2.6 wt. $\% \mathrm{PbO}(0.02$ to 0.03 apfu $\mathrm{Pb})$. Small amounts of REE and Y were detected in uraninite: $\sum$ REE $+Y$ oxide values attain 2.3 to 3.4 wt. \% (0.04-0.05 apfu REE+Y). The HREE (Gd to Lu) +Y slightly prevail over LREE (La to $\mathrm{Eu}$ ) contents; they achieve 51 to 67 at. \% (57 at. \% on average) of $\sum \mathrm{REE}+\mathrm{Y}$ concentration. Among them, $\mathrm{Y}$
Tab. 3 Chemical composition of uraninite (in wt. \% and apfu)

\begin{tabular}{|c|c|c|c|c|c|c|c|}
\hline & 1 & 2 & 3 & 3 & 5 & 6 & 7 \\
\hline $\mathrm{P}_{2} \mathrm{O}_{5}$ & 0.09 & 0.08 & 0.06 & 0.04 & 0.08 & 0.04 & 0.06 \\
\hline $\mathrm{As}_{2} \mathrm{O}_{5}$ & 0.12 & 0.13 & 0.13 & 0.12 & 0.12 & 0.12 & 0.10 \\
\hline $\mathrm{SiO}_{2}$ & 0.06 & 0.12 & 0.06 & 0.04 & 0.05 & 0.03 & 0.06 \\
\hline $\mathrm{UO}_{2}$ & 92.11 & 91.40 & 92.44 & 92.04 & 92.49 & 91.97 & 92.90 \\
\hline $\mathrm{Y}_{2} \mathrm{O}_{3}$ & 0.34 & 0.34 & 0.22 & 0.29 & 0.36 & 0.25 & 0.17 \\
\hline $\mathrm{Ce}_{2} \mathrm{O}_{3}$ & 0.13 & 0.15 & 0.15 & 0.16 & 0.15 & 0.14 & 0.10 \\
\hline $\mathrm{Pr}_{2} \mathrm{O}_{3}$ & 0.33 & 0.32 & 0.28 & 0.34 & 0.30 & 0.29 & 0.34 \\
\hline $\mathrm{Nd}_{2} \mathrm{O}_{3}$ & 0.17 & 0.18 & 0.11 & 0.17 & 0.14 & 0.17 & 0.04 \\
\hline $\mathrm{Sm}_{2} \mathrm{O}_{3}$ & 0.31 & 0.40 & 0.27 & 0.35 & 0.40 & 0.37 & 0.30 \\
\hline $\mathrm{Eu}_{2} \mathrm{O}_{3}$ & 0.27 & 0.24 & 0.24 & 0.30 & 0.24 & 0.24 & 0.28 \\
\hline $\mathrm{Gd}_{2} \mathrm{O}_{3}$ & 0.23 & 0.28 & 0.17 & 0.20 & 0.23 & 0.16 & 0.15 \\
\hline $\mathrm{Tb}_{2} \mathrm{O}_{3}$ & 0.09 & 0.10 & 0.08 & 0.13 & 0.10 & 0.11 & 0.00 \\
\hline $\mathrm{Dy}_{2} \mathrm{O}_{3}$ & 0.27 & 0.28 & 0.16 & 0.15 & 0.31 & 0.13 & 0.15 \\
\hline $\mathrm{Ho}_{2} \mathrm{O}_{3}$ & 0.00 & 0.00 & 0.00 & 0.05 & 0.07 & 0.00 & 0.06 \\
\hline $\mathrm{Er}_{2} \mathrm{O}_{3}$ & 0.55 & 0.59 & 0.54 & 0.54 & 0.61 & 0.56 & 0.51 \\
\hline $\mathrm{Tm}_{2} \mathrm{O}_{3}$ & 0.10 & 0.04 & 0.11 & 0.12 & 0.13 & 0.11 & 0.16 \\
\hline $\mathrm{Yb}_{2} \mathrm{O}_{3}$ & 0.24 & 0.27 & 0.22 & 0.22 & 0.31 & 0.19 & 0.21 \\
\hline $\mathrm{Lu}_{2} \mathrm{O}_{3}$ & 0.11 & 0.12 & 0.11 & 0.10 & 0.08 & 0.12 & 0.09 \\
\hline $\mathrm{CaO}$ & 0.27 & 0.30 & 0.28 & 0.32 & 0.24 & 0.53 & 0.32 \\
\hline $\mathrm{PbO}$ & 2.28 & 2.54 & 2.44 & 2.45 & 2.18 & 2.61 & 2.56 \\
\hline Total & 98.07 & 97.88 & 98.07 & 98.13 & 98.59 & 98.14 & 98.56 \\
\hline $\mathrm{P}$ & 0.003 & 0.003 & 0.002 & 0.001 & 0.003 & 0.001 & 0.002 \\
\hline As & 0.003 & 0.003 & 0.003 & 0.003 & 0.003 & 0.003 & 0.002 \\
\hline $\mathrm{Si}$ & 0.003 & 0.005 & 0.003 & 0.002 & 0.002 & 0.001 & 0.003 \\
\hline $\mathrm{U}$ & 0.903 & 0.893 & 0.909 & 0.902 & 0.902 & 0.895 & 0.909 \\
\hline $\mathrm{Y}$ & 0.008 & 0.008 & 0.005 & 0.007 & 0.008 & 0.006 & 0.004 \\
\hline $\mathrm{Ce}$ & 0.002 & 0.002 & 0.002 & 0.003 & 0.002 & 0.002 & 0.002 \\
\hline $\operatorname{Pr}$ & 0.005 & 0.005 & 0.005 & 0.005 & 0.005 & 0.005 & 0.005 \\
\hline $\mathrm{Nd}$ & 0.003 & 0.003 & 0.002 & 0.003 & 0.002 & 0.003 & 0.001 \\
\hline $\mathrm{Sm}$ & 0.005 & 0.006 & 0.004 & 0.005 & 0.006 & 0.006 & 0.005 \\
\hline $\mathrm{Eu}$ & 0.004 & 0.004 & 0.004 & 0.005 & 0.004 & 0.004 & 0.004 \\
\hline $\mathrm{Gd}$ & 0.003 & 0.004 & 0.002 & 0.003 & 0.003 & 0.002 & 0.002 \\
\hline $\mathrm{Tb}$ & 0.001 & 0.001 & 0.001 & 0.002 & 0.001 & 0.002 & 0,000 \\
\hline Dy & 0.004 & 0.004 & 0.002 & 0.002 & 0.004 & 0.002 & 0.002 \\
\hline Ho & 0,000 & 0,000 & 0,000 & 0.001 & 0.001 & 0,000 & 0.001 \\
\hline $\mathrm{Er}$ & 0.008 & 0.008 & 0.007 & 0.007 & 0.008 & 0.008 & 0.007 \\
\hline $\mathrm{Tm}$ & 0.001 & 0.001 & 0.002 & 0.002 & 0.002 & 0.001 & 0.002 \\
\hline $\mathrm{Yb}$ & 0.003 & 0.004 & 0.003 & 0.003 & 0.004 & 0.003 & 0.003 \\
\hline $\mathrm{Lu}$ & 0.001 & 0.002 & 0.001 & 0.001 & 0.001 & 0.002 & 0.001 \\
\hline $\mathrm{Ca}$ & 0.013 & 0.014 & 0.013 & 0.015 & 0.011 & 0.025 & 0.015 \\
\hline $\mathrm{Pb}$ & 0.027 & 0.030 & 0.029 & 0.029 & 0.026 & 0.031 & 0.030 \\
\hline Sum cat. & 1.000 & 1.000 & 0.999 & 1.001 & 0.998 & 1.002 & 1.000 \\
\hline $\mathrm{O}$ & 1.939 & 1.933 & 1.940 & 1.934 & 1.939 & 1.925 & 1.938 \\
\hline
\end{tabular}

Formulae based on sum of cations $=1$ apfu

Th contents are below the detection limit and $\mathrm{Er}$ are the most abundant (10-20 and 14-19 at. \% of $\sum$ REE + Y). A negative REE+Y vs. U correlation (Fig. $5)$ indicates some isomorphic substitution mechanisms, such as $(\mathrm{REE}, \mathrm{Y})^{3+}+(\mathrm{OH})^{-}=\mathrm{U}^{4+}+\mathrm{O}^{2-}$ or $4(\mathrm{REE}, \mathrm{Y})^{3+}=$ $3 \mathrm{U}^{4+}+\square$. However, a possible presence of uranium also in $\mathrm{U}^{6+}$ form together with negligible contents of $\mathrm{Ca}, \mathrm{P}$, As, and Si detected in uraninite (Tab. 3) could be, in part, contributing to other substitutions. 


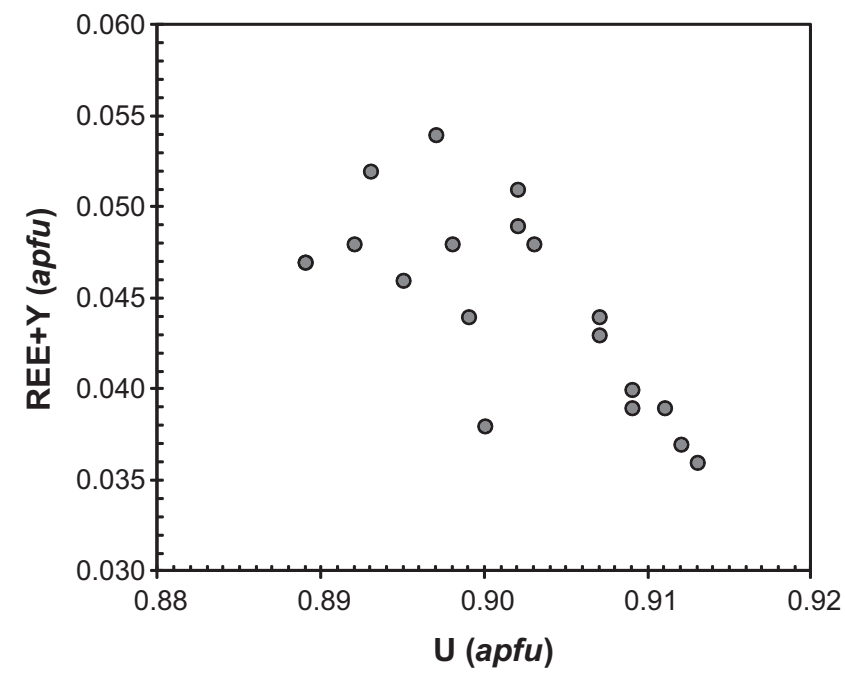

Fig. 5 Chemical composition of uraninite from Čučma in REE+Y vs. U substitution diagram (apfu).

\subsection{Florencite-(Ce) and goyazite}

Florencite-(Ce), ideally $\mathrm{CeAl}_{3}\left(\mathrm{PO}_{4}\right)_{2}(\mathrm{OH})_{6}$, was identified as irregular aggregates, veinlets and fillings of fractures in fluorapatite crystals (Fig. 6a). Goyazite, ideally $\mathrm{SrAl}_{3}\left(\mathrm{PO}_{4}\right)_{2}(\mathrm{OH})_{6}$, occurs as chaotic veinlets and fracture fillings of fluorapatite crystals. It commonly shows an irregular chemical zoning (Fig. 6b), which is caused by variable content of REE. Both minerals represent supergene products of monazite-(Ce) or monazite-(Nd) alteration and are often associated with other supergene phases, like autunite to meta-autunite, goethite, opal, torbernite and unaltered relicts of primary monazite-(Ce), uraninite, and xenotime-(Y). Representative EMP analyses of florencite-(Ce) and goyazite from Čučma are compiled in Tab. 4. Florencite-(Ce) to goyazite contains low to moderate concentrations of $\mathrm{Sr}(5.2-11.7$ wt. \% $\mathrm{SrO}$; 0.25-0.55 apfu Sr) and very low concentrations of As (up to 0.15 wt. $\% \mathrm{As}_{2} \mathrm{O}_{5} ; 0.001$ apfu As), which reflects only a minor miscibility in the system $(\mathrm{Ce}, \mathrm{Sr}) \mathrm{Al}_{3}\left(\mathrm{PO}_{4}\right)_{2}(\mathrm{OH})_{6-x}$ $\left(\mathrm{H}_{2} \mathrm{O}\right)_{x}-(\mathrm{Ce}, \mathrm{Sr}) \mathrm{Al}_{3}\left(\mathrm{AsO}_{4}\right)_{2}(\mathrm{OH})_{6-x}\left(\mathrm{H}_{2} \mathrm{O}\right)_{x}$ (Fig. 7a). The presence of low concentrations of $\mathrm{S}\left(0.1-0.5 \mathrm{wt}\right.$. $\% \mathrm{SO}_{3}$; 0.008-0.03 apfu S) may be attributed to the substitution $\mathrm{Sr}^{2+}+\mathrm{S}^{6+}=\mathrm{Ce}^{3+}+\mathrm{P}^{5+}$, reflecting a subordinate presence of the svanbergite, $\mathrm{SrAl}_{3}\left[(\mathrm{OH})_{6}\left(\mathrm{SO}_{4}\right)\left(\mathrm{PO}_{4}\right)\right]$, component (Fig. 7b). The concentrations of actinide elements (Th, $\mathrm{U})$ are generally low, not exceeding 0.5 wt. \% of each oxide. Lead occurs in variable amounts, up to $3.6 \mathrm{wt}$ \% $\mathrm{PbO}(0.09$ apfu $\mathrm{Pb})$. It may be preferentially coordinated with $\mathrm{Fe}^{3+}$ (assuming that all $\mathrm{Fe}$ is trivalent), as kintoreite, $\mathrm{PbFe}_{3}\left[(\mathrm{OH})_{6}\left(\mathrm{PO}_{3} \mathrm{OH}\right)\left(\mathrm{PO}_{4}\right)\right]$. In some cases, $\mathrm{Pb}(a p f u$ ) slightly exceeds Fe (apfu), possibly implying only a limited solid solution toward plumbogummite, $\mathrm{PbAl}_{3}\left[(\mathrm{OH})_{6}\left(\mathrm{PO}_{3} \mathrm{OH}\right)\left(\mathrm{PO}_{4}\right)\right]$. Contents of $\mathrm{Ca}$, ranging from 0.5 to 2.0 wt. $\% \mathrm{CaO}(0.04-0.18$ apfu $\mathrm{Ca})$ suggest also an influence of crandallite-type substitution mechanism (Fig. 8). Florencite-(Ce) and goyazite contains up to 0.43 wt. \% F, substituting for $\mathrm{OH}(0.09$ apfu $\mathrm{F})$.

\subsection{Chemical $U-P b$ dating of uraninite}

The chemical, in situ electron-microprobe U-Pb dating of uraninite (20 point analyses) gave an age interval between 171 and $215 \pm 2 \mathrm{Ma}$ according to Montel et al. (1996) calculation (Tab. 5). Histogram shows 191 to $220 \mathrm{Ma}$ as a main age interval with three scattered younger age determinations (Fig. 9). Consequently, the main uraninite age population shows the average value of $207 \pm 2 \mathrm{Ma}(\mathrm{n}=$ 17, 26). The calculated ages (after Montel et al. 1996) are almost identical in comparison to those obtained by the procedure of Bowles (1990). The empirical expressions by Ranchin (1968) and by Cameron-Schiman (1978) gave $1-1.5$ and $7-7.5 \%$ higher ages, respectively, compared to Montel et al. (1996) calculation (Tab. 5, Fig. 10).

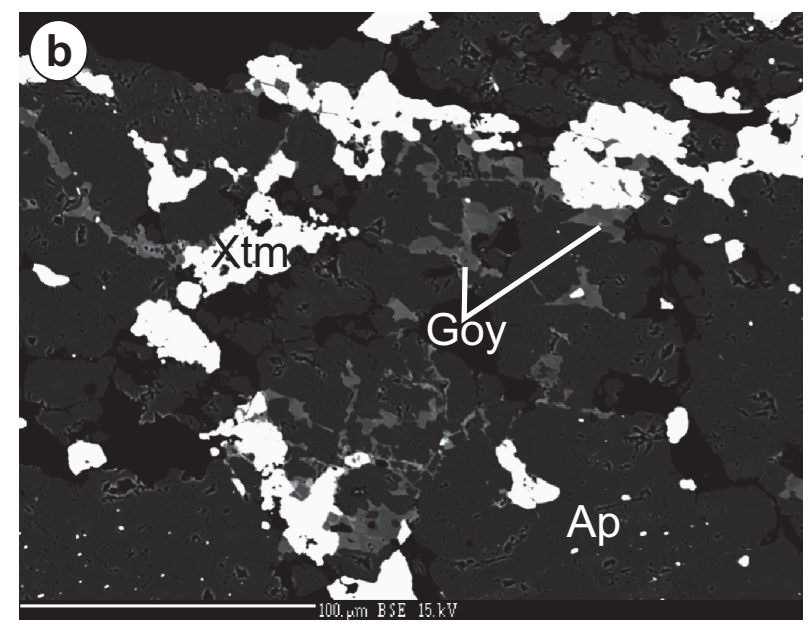

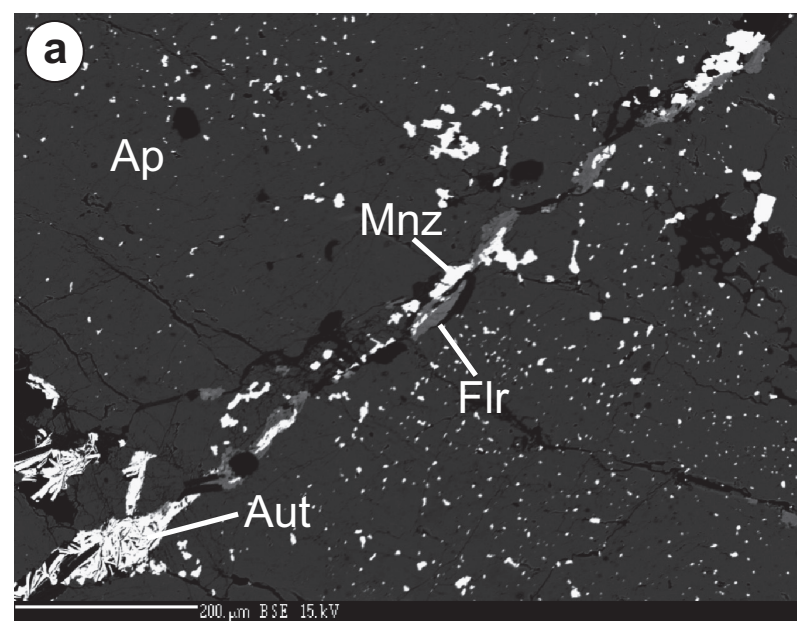

Fig. 6a - Irregular aggregates and fillings of florencite-(Ce) (Flr) associated with autunite (Aut) and altered monazite-(Ce) (Mnz) in fracture between two fluorapatite crystals (Ap), BEI. b - Goyazite fracture fillings (Goy) together with xenotime-(Y) aggregates (Xtm) in fluorapatite (Ap), BEI. 
Tab. 4 Representative analyses of florencite-(Ce) and goyazite (in wt. \% and apfu)

\begin{tabular}{|c|c|c|c|c|c|c|c|c|c|c|}
\hline & $\begin{array}{c}\text { florencite- } \\
-(\mathrm{Ce})\end{array}$ & $\begin{array}{c}\text { florencite- } \\
-(\mathrm{Ce})\end{array}$ & $\begin{array}{c}\text { florencite- } \\
-(\mathrm{Ce})\end{array}$ & $\begin{array}{c}\text { florencite- } \\
-(\mathrm{Ce})\end{array}$ & $\begin{array}{l}\text { florencite- } \\
-(\mathrm{Ce})\end{array}$ & $\begin{array}{l}\text { florencite- } \\
-(\mathrm{Ce})\end{array}$ & goyazite & goyazite & goyazite & goyazite \\
\hline $\mathrm{SO}_{3}$ & 0.27 & 0.14 & 0.39 & 0.14 & 0.54 & 0.16 & 0.16 & 0.13 & 0.27 & 0.08 \\
\hline $\mathrm{P}_{2} \mathrm{O}_{5}^{3}$ & 26.40 & 26.69 & 26.89 & 25.76 & 24.59 & 26.73 & 26.85 & 27.83 & 27.96 & 28.84 \\
\hline $\mathrm{As}_{2} \mathrm{O}_{5}$ & 0.14 & 0.15 & 0.07 & 0.13 & 0.14 & 0.08 & 0.13 & 0.00 & 0.08 & 0.13 \\
\hline $\mathrm{SiO}_{2}^{2}$ & 0.09 & 0.09 & 0.25 & 0.33 & 1.22 & 0.06 & 0.14 & 0.09 & 0.03 & 0.06 \\
\hline $\mathrm{ThO}_{2}^{2}$ & 0.00 & 0.01 & 0.00 & 0.01 & 0.01 & 0.00 & 0.01 & 0.02 & 0.02 & 0.00 \\
\hline $\mathrm{UO}_{2}{ }^{2}$ & 0.18 & 0.06 & 0.00 & 0.43 & 0.19 & 0.00 & 0.12 & 0.04 & 0.00 & 0.07 \\
\hline $\mathrm{ZrO}_{2}^{2}$ & 0.10 & 0.08 & 0.01 & 0.08 & 0.05 & 0.09 & 0.12 & 0.06 & 0.06 & 0.08 \\
\hline $\mathrm{HfO}_{2}^{2}$ & 0.07 & 0.00 & 0.06 & 0.00 & 0.01 & 0.00 & 0.00 & 0.00 & 0.05 & 0.10 \\
\hline $\mathrm{Al}_{2} \mathrm{O}_{3}^{2}$ & 30.54 & 31.00 & 30.87 & 29.73 & 30.07 & 29.52 & 29.98 & 31.41 & 31.18 & 30.97 \\
\hline $\mathrm{Fe}_{2} \mathrm{O}_{3}^{3}$ & 0.88 & 1.42 & 0.11 & 0.91 & 0.73 & 0.42 & 1.83 & 0.57 & 0.15 & 0.21 \\
\hline $\mathrm{Y}_{2} \mathrm{O}_{3}^{3}$ & 0.00 & 0.01 & 0.00 & 0.01 & 0.04 & 0.00 & 0.00 & 0.01 & 0.02 & 0.00 \\
\hline $\mathrm{La}_{2} \mathrm{O}_{3}^{3}$ & 3.09 & 2.84 & 3.56 & 2.31 & 3.57 & 2.58 & 1.97 & 2.93 & 3.39 & 1.69 \\
\hline $\mathrm{Ce}_{2}^{2} \mathrm{O}_{3}^{3}$ & 8.85 & 8.58 & 9.69 & 6.70 & 8.48 & 8.35 & 5.57 & 7.24 & 7.26 & 5.37 \\
\hline $\mathrm{Pr}_{2} \mathrm{O}_{3}$ & 1.47 & 1.39 & 1.58 & 1.26 & 1.23 & 1.56 & 0.91 & 0.99 & 0.92 & 0.96 \\
\hline $\mathrm{Nd}_{2}^{2} \mathrm{O}_{3}^{3}$ & 6.68 & 6.46 & 7.67 & 7.26 & 4.87 & 7.49 & 3.90 & 3.47 & 3.17 & 4.87 \\
\hline $\mathrm{Sm}_{2} \mathrm{O}_{3}$ & 1.82 & 1.71 & 1.43 & 2.74 & 1.14 & 2.25 & 0.80 & 0.56 & 0.36 & 1.43 \\
\hline $\mathrm{Eu}_{2} \mathrm{O}_{3}{ }_{3}^{3}$ & 0.20 & 0.10 & 0.04 & 0.23 & 0.10 & 0.16 & 0.14 & 0.07 & 0.09 & 0.11 \\
\hline $\mathrm{Gd}_{2}^{2} \mathrm{O}_{3}^{3}$ & 0.67 & 0.36 & 0.16 & 0.46 & 0.21 & 0.40 & 0.13 & 0.15 & 0.03 & 0.49 \\
\hline $\mathrm{Tb}_{2}^{2} \mathrm{O}_{3}^{3}$ & 0.03 & 0.02 & 0.03 & 0.00 & 0.03 & 0.00 & 0.10 & 0.04 & 0.00 & 0.01 \\
\hline $\mathrm{Dy}_{2}^{2} \mathrm{O}_{3}^{3}$ & 0.00 & 0.06 & 0.00 & 0.06 & 0.02 & 0.06 & 0.02 & 0.00 & 0.07 & 0.03 \\
\hline $\mathrm{Ho}_{2} \mathrm{O}_{3}^{3}$ & 0.00 & 0.00 & 0.00 & 0.05 & 0.00 & 0.01 & 0.00 & 0.05 & 0.00 & 0.02 \\
\hline $\mathrm{Er}_{2} \mathrm{O}_{3}{ }^{3}$ & 0.21 & 0.07 & 0.27 & 0.28 & 0.27 & 0.13 & 0.22 & 0.13 & 0.26 & 0.13 \\
\hline $\mathrm{Tm}_{2}^{2} \mathrm{O}_{3}^{3}$ & 0.01 & 0.06 & 0.00 & 0.01 & 0.03 & 0.04 & 0.03 & 0.08 & 0.05 & 0.00 \\
\hline $\mathrm{Yb}_{2} \mathrm{O}_{3}^{3}$ & 0.07 & 0.11 & 0.11 & 0.06 & 0.05 & 0.10 & 0.08 & 0.06 & 0.06 & 0.09 \\
\hline $\mathrm{Lu}_{2} \mathrm{O}_{3}$ & 0.12 & 0.00 & 0.00 & 0.15 & 0.03 & 0.06 & 0.09 & 0.05 & 0.00 & 0.03 \\
\hline $\mathrm{CaO}^{2}$ & 0.47 & 1.15 & 0.61 & 1.18 & 1.10 & 2.02 & 1.36 & 0.66 & 0.65 & 1.03 \\
\hline $\mathrm{SrO}$ & 6.04 & 6.59 & 5.24 & 6.28 & 6.71 & 5.45 & 11.18 & 10.98 & 11.74 & 11.69 \\
\hline $\mathrm{PbO}$ & 0.10 & 0.23 & 0.024 & 2.59 & 1.08 & 0.09 & 0.23 & 0.01 & 0.02 & 0.12 \\
\hline $\mathrm{F}$ & 0.00 & 0.00 & 0.00 & 0.01 & 0.00 & 0.00 & 0.33 & 0.23 & 0.43 & 0.22 \\
\hline $\mathrm{Cl}$ & 0.02 & 0.03 & 0.03 & 0.02 & 0.03 & 0.02 & 0.02 & 0.01 & 0.01 & 0.02 \\
\hline $\mathrm{H}_{2} \mathrm{O}$ calc. & 11.42 & 11.88 & 11.40 & 11.63 & 11.56 & 11.51 & 11.94 & 11.98 & 11.94 & 12.04 \\
\hline Total & 88.46 & 89.38 & 89.12 & 89.16 & 86.49 & 87.81 & 86.40 & 87.83 & 88.30 & 88.83 \\
\hline $\mathrm{O}=\mathrm{F}$ & 0.00 & 0.00 & 0.00 & -0.00 & 0.00 & 0.00 & -0.14 & -0.10 & -0.18 & -0.09 \\
\hline $\mathrm{O}=\mathrm{Cl}$ & -0.00 & -0.01 & -0.01 & -0.01 & -0.01 & -0.01 & -0.00 & -0.00 & -0.00 & -0.01 \\
\hline Total & 99.88 & 101.25 & 100.51 & 100.78 & 98.04 & 99.31 & 98.20 & 99.72 & 100.05 & 100.77 \\
\hline $\mathrm{S}^{6+}$ & 0.017 & 0.009 & 0.024 & 0.009 & 0.034 & 0.010 & 0.010 & 0.008 & 0.016 & 0.005 \\
\hline $\mathrm{P}^{5+}$ & 1.868 & 1.846 & 1.887 & 1.831 & 1.755 & 1.896 & 1.873 & 1.910 & 1.919 & 1.961 \\
\hline $\mathrm{As}^{5+}$ & 0.006 & 0.007 & 0.003 & 0.006 & 0.006 & 0.004 & 0.005 & 0.000 & 0.003 & 0.006 \\
\hline $\mathrm{Si}^{4+}$ & 0.008 & 0.007 & 0.020 & 0.027 & 0.102 & 0.005 & 0.012 & 0.007 & 0.002 & 0.005 \\
\hline $\mathrm{Th}^{4+}$ & 0.000 & 0.000 & 0.000 & 0.000 & 0.000 & 0.000 & 0.000 & 0.000 & 0.000 & 0.000 \\
\hline $\mathrm{U}^{4+}$ & 0.003 & 0.001 & 0.000 & 0.008 & 0.004 & 0.000 & 0.002 & 0.001 & 0.000 & 0.001 \\
\hline $\mathrm{Fe}^{3+}$ & 0.055 & 0.087 & 0.007 & 0.058 & 0.046 & 0.026 & 0.113 & 0.035 & 0.009 & 0.012 \\
\hline $\mathrm{Zr}^{4+}$ & 0.003 & 0.002 & 0.003 & 0.002 & 0.001 & 0.003 & 0.004 & 0.002 & 0.002 & 0.002 \\
\hline $\mathrm{Hf}^{4+}$ & 0.001 & 0.000 & 0.002 & 0.000 & 0.000 & 0.000 & 0.000 & 0.000 & 0.001 & 0.002 \\
\hline $\mathrm{Al}^{3+}$ & 3.009 & 2.984 & 3.015 & 2.942 & 2.988 & 2.914 & 2.911 & 3.001 & 2.979 & 2.933 \\
\hline $\mathrm{Y}^{3+}$ & 0.000 & 0.000 & 0.000 & 0.000 & 0.002 & 0.000 & 0.000 & 0.000 & 0.001 & 0.000 \\
\hline $\mathrm{La}^{3+}$ & 0.095 & 0.086 & 0.109 & 0.071 & 0.111 & 0.080 & 0.060 & 0.087 & 0.101 & 0.050 \\
\hline $\mathrm{Ce}^{3+}$ & 0.271 & 0.257 & 0.294 & 0.206 & 0.262 & 0.256 & 0.168 & 0.215 & 0.215 & 0.158 \\
\hline $\operatorname{Pr}^{3+}$ & 0.045 & 0.041 & 0.048 & 0.039 & 0.038 & 0.048 & 0.027 & 0.029 & 0.027 & 0.028 \\
\hline $\mathrm{Nd}^{3+}$ & 0.199 & 0.188 & 0.227 & 0.218 & 0.147 & 0.224 & 0.115 & 0.100 & 0.092 & 0.140 \\
\hline $\mathrm{Sm}^{3+}$ & 0.053 & 0.048 & 0.041 & 0.079 & 0.033 & 0.065 & 0.023 & 0.016 & 0.001 & 0.040 \\
\hline $\mathrm{Gd}^{3+}$ & 0.019 & 0.010 & 0.004 & 0.013 & 0.006 & 0.011 & 0.004 & 0.004 & 0.001 & 0.013 \\
\hline $\mathrm{Tb}^{3+}$ & 0.001 & 0.001 & 0.001 & 0.000 & 0.001 & 0.000 & 0.003 & 0.001 & 0.000 & 0.000 \\
\hline $\mathrm{Dy}^{3+}$ & 0.000 & 0.002 & 0.000 & 0.002 & 0.001 & 0.002 & 0.001 & 0.000 & 0.002 & 0.001 \\
\hline $\mathrm{Ho}^{3+}$ & 0.000 & 0.000 & 0,000 & 0.001 & 0.000 & 0.000 & 0.000 & 0.001 & 0.000 & 0.000 \\
\hline $\mathrm{Er}^{3+}$ & 0.005 & 0.002 & 0.007 & 0.007 & 0.007 & 0.003 & 0.006 & 0.003 & 0.007 & 0.003 \\
\hline $\mathrm{Tm}^{3+}$ & 0.000 & 0.002 & 0.000 & 0.000 & 0.001 & 0.001 & 0.001 & 0.002 & 0.001 & 0.000 \\
\hline $\mathrm{Yb}^{3+}$ & 0.002 & 0.003 & 0.003 & 0.002 & 0.001 & 0.003 & 0.002 & 0.002 & 0.001 & 0.002 \\
\hline $\mathrm{Lu}^{3+}$ & 0.003 & 0.000 & 0.000 & 0.004 & 0.001 & 0.001 & 0.002 & 0.001 & 0.000 & 0.001 \\
\hline $\mathrm{Ca}^{2+}$ & 0.042 & 0.101 & 0.054 & 0.106 & 0.100 & 0.181 & 0.120 & 0.057 & 0.056 & 0.089 \\
\hline $\mathrm{Sr}^{2+}$ & 0.293 & 0.312 & 0.252 & 0.306 & 0.328 & 0.265 & 0.534 & 0.516 & 0.552 & 0.545 \\
\hline $\mathrm{Pb}^{2+}$ & 0.002 & 0.005 & 0.001 & 0.061 & 0.026 & 0.002 & 0.005 & 0.000 & 0.001 & 0.003 \\
\hline $\mathrm{F}^{-}$ & 0.000 & 0.000 & 0.000 & 0.001 & 0.000 & 0.000 & 0.085 & 0.058 & 0.110 & 0.055 \\
\hline $\mathrm{Cl}^{-}$ & 0.003 & 0.004 & 0.005 & 0.003 & 0.005 & 0.003 & 0.003 & 0.001 & 0.001 & 0.003 \\
\hline Charge Balance & 21.478 & 21.323 & 21.571 & 21.266 & 21.278 & 21.389 & 21.144 & 21.281 & 21.291 & 21.322 \\
\hline $\mathrm{OH}^{-}$ & 0.519 & 0.672 & 0.424 & 0.730 & 0.717 & 0.608 & 0.767 & 0.660 & 0.598 & 0.619 \\
\hline $\mathrm{OH}$ total $-\mathrm{F}-\mathrm{Cl}$ & 6.519 & 6.672 & 6.424 & 6.730 & 6.717 & 6.608 & 6.767 & 6.660 & 6.598 & 6.619 \\
\hline $\mathrm{OH}$ total $+\mathrm{F}+\mathrm{Cl}$ & 6.522 & 6.677 & 6.429 & 6.734 & 6.722 & 6.611 & 6.856 & 6.719 & 6.709 & 6.678 \\
\hline $\mathrm{O}=4-\mathrm{OH}$ & 3.478 & 3.323 & 3.571 & 3.266 & 3.278 & 3.389 & 3.144 & 3.281 & 3.291 & 3.322 \\
\hline $\mathrm{O}$ & 7.478 & 7.323 & 7.571 & 7.266 & 7.278 & 7.389 & 7.144 & 7.281 & 7.291 & 7.322 \\
\hline $\mathrm{O}_{\text {total }}$ & 14.000 & 14.000 & 14.000 & 14.000 & 14.000 & 14.000 & 14.000 & 14.000 & 14.000 & 14.000 \\
\hline position $\mathrm{M}^{2+}+\mathrm{REE}^{3+}$ & 1.029 & 1.057 & 1.040 & 1.116 & 1.063 & 1.142 & 1.069 & 1.036 & 1.067 & 1.073 \\
\hline position $\mathrm{Al}^{3+}, \mathrm{Fe}^{3+}$ & 3.064 & 3.071 & 3.022 & 3.000 & 3.034 & 2.940 & 3.025 & 3.036 & 2.989 & 2.945 \\
\hline position $\mathrm{X}$ & 1.899 & 1.868 & 1.935 & 1.874 & 1.897 & 1.915 & 1.900 & 1.925 & 1.941 & 1.977 \\
\hline
\end{tabular}

Formulae based on 6 cations and 14 oxygen atoms 
(a)

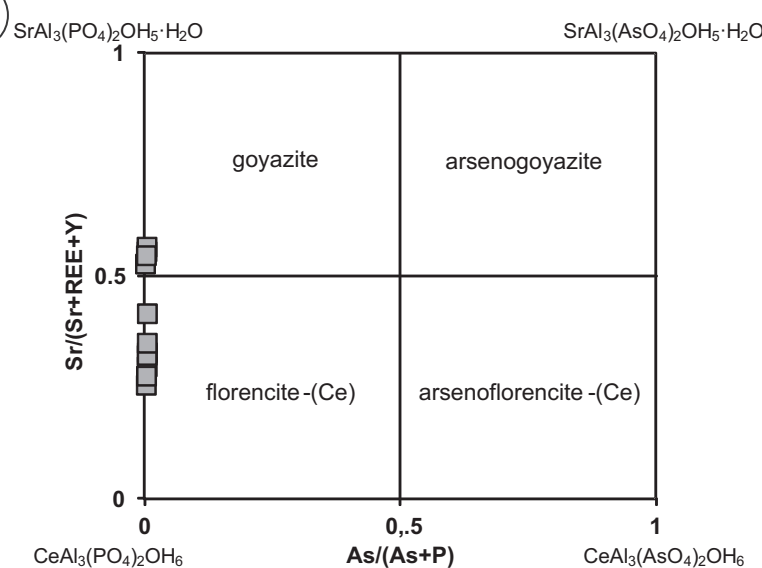

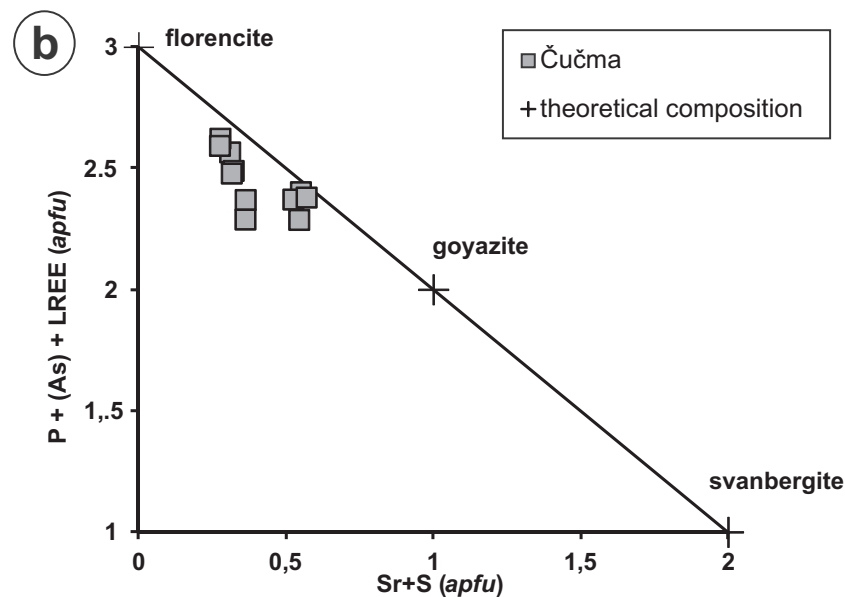

Fig. 7 Compositions of florencite-(Ce) and goyazite from Čučma. a - Quadrilateral classification diagram of the $(\mathrm{Sr}+\mathrm{REE}+\mathrm{Y}) \mathrm{PO}-(\mathrm{Sr}+\mathrm{REE}$ $+\mathrm{Y}) \mathrm{AsO}_{4}$ system (atomic proportions). b - Binary plot P + As + LREE vs. $\mathrm{Sr}+\mathrm{S}(a p f u)$.

\section{Discussion and conclusions}

\subsection{Age of REE-U mineralization}

Four different calculation procedures were applied to determine an age of uraninite from the Čučma REE-U vein mineralization. The most matching results gave approaches of Bowles (1990) and Montel et al. (1996). These two methods use more precise equations including decay constants in contrast to older approximate empirical formulae (Ranchin 1968; Cameron-Schiman 1978) and thus are preferred here.

The chemical (electron-microprobe) dating of uraninite indicates a Late Triassic, Rhaetian age of the Čučma REE$\mathrm{U}$ mineralization with an average value of $207 \pm 2 \mathrm{Ma}$ (n $=17,2 \sigma)$. Scattered younger age results (171 to $193 \pm 2$ Ma) come from smaller uraninite grains $(<10 \mu \mathrm{m}$ across $)$ and most probably they reflect an episodic $\mathrm{Pb}$ loss by some

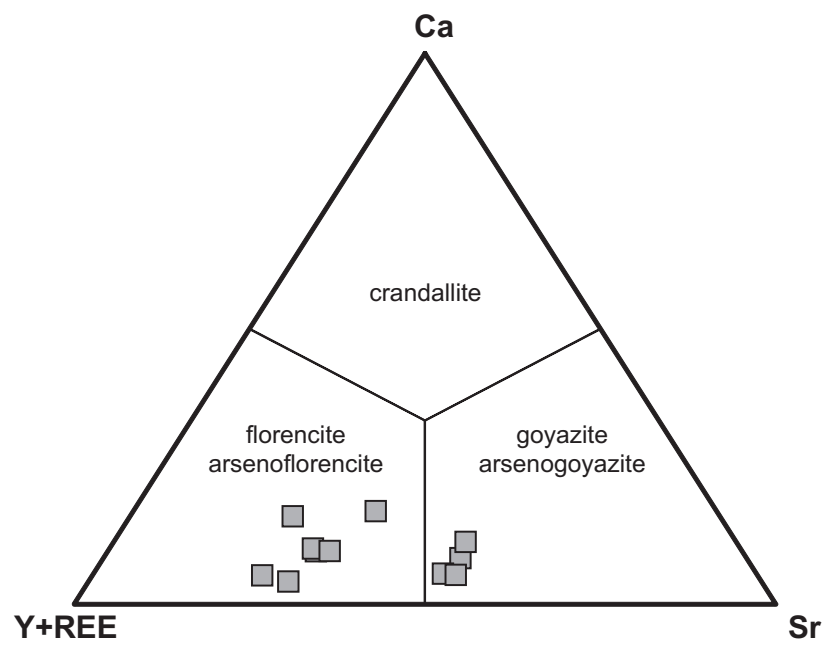

Fig. 8 Compositions of florencite-(Ce) and goyazite from Čučma in ternary classification $\mathrm{REE}+\mathrm{Y}-\mathrm{Ca}-\mathrm{Sr}$ diagram $(a p f u)$. younger thermal overprinting and/or recrystallization event. Such an overprint would be connected with partial alteration of uraninite, fluorapatite, monazite, and xenotime and formation of florencite-(Ce), goyazite and other supergene phases. Consequently, we can assume the Late Triassic $(\sim 205 \mathrm{Ma})$ as the most probable age of precipitation of uraninite and the primary REE-U mineralization near Čučma.

The age of uraninite from the Čučma REE-U mineralization is distinctly younger than solidification of adjacent Permian granites of the Spiš-Gemer type and associated $\mathrm{Sn}-\mathrm{W}-\mathrm{Mo}$ mineralization in the Gemeric Superunit (c. 270 to $250 \mathrm{Ma}$ : U-Pb zircon dating, Poller et al. 2002 and Re-Os molybdenite age, Kohút and Stein 2005, and references therein). Similar ages ( $\sim 275$ to 265 Ma) yielded zircon SHRIMP U-Pb dating of volcanic rocks (basaltic andesites and rhyodacites) from the North

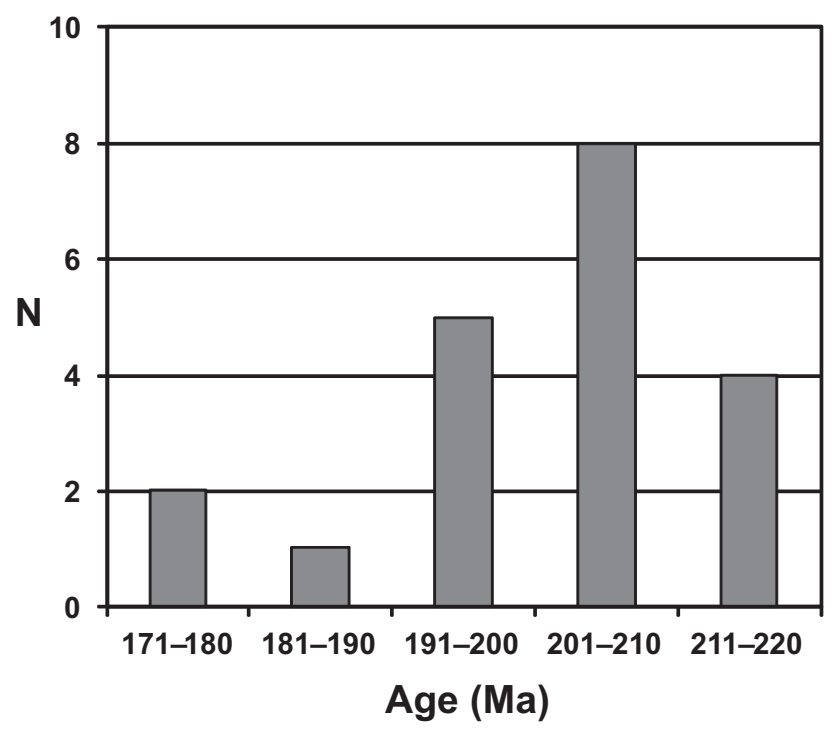

Fig. 9 Histogram of uraninite ages from Čučma, based on Montel et al. (1996) calculation. 


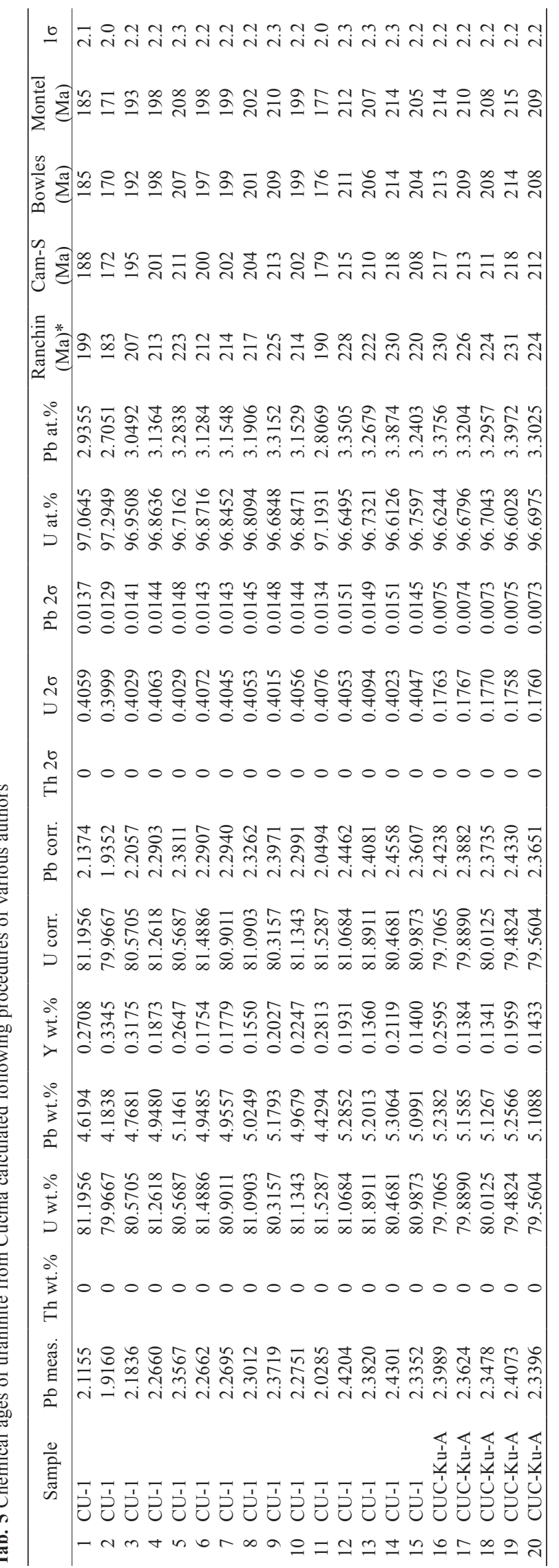

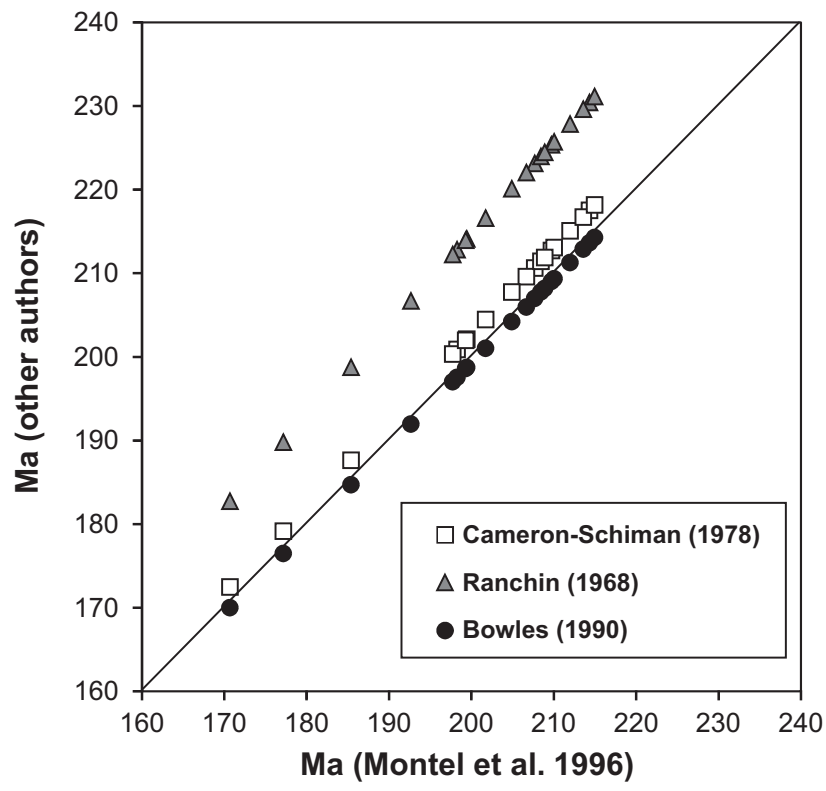

Fig. 10 Comparison of uraninite chemical ages (in Ma) from Čučma calculated according to various authors (Ranchin 1968; Cameron-Schiman 1978; Bowles 1990; Montel et al. 1996). The solid line represents an ideal (1:1) correlation between the Montel et al. (1996) and other calculation approaches.

Gemeric Unit and the Bôrka Nappe, both also in the Gemeric Superunit (Vozárová et al. 2012). Consequently, the direct genetic link between the Permian granites or volcanic rocks and the Čučma vein mineralization is not likely. On the other hand, there are several younger, Late Triassic to Early Jurassic age determinations ( $~ 195$ to $210 \mathrm{Ma}$ ) in the Gemeric Superunit, analogous to the Čučma REE-U mineralization. They include muscovite (fuchsite) $\mathrm{K}-\mathrm{Ar}$ dating from siderite hydrothermal vein at Rudňany (205 Ma; Bagdasaryan et al. 1977; Cambel et al. 1990), and muscovite K-Ar dating of the Hnilec granite (195 to $210 \mathrm{Ma}$; Cambel et al. 1990). An early Alpine heating event between 225 to $205 \mathrm{Ma}$ is indicated by some monazite ages of metatectic granite from Rožňava, Gul'apalag (U-Th-Pb chemical dating; Radvanec et al. 2009). Recent Re-Os molybdenite dating of the Kurišková uranium deposit near Košice gave Late Permian ages $(257.2 \pm 3.0 \mathrm{Ma}$ to $255.6 \pm 3.7 \mathrm{Ma})$ for massive vein mineralization originated from igneous source (Kohút et al. 2013), whereas the superimposed U remobilization within shear zones occurred in Triassic-Jurassic ( 200-160 Ma) as documented by chemical dating of uraninite (Demko et al. 2011, 2012).

On the basis of structural data, the Late Triassic was characterized by an early Alpine extension, following unroofing of the Paleozoic basement rocks of the Gemeric Superunit ( $\mathrm{VD}_{2}$ stage, Grecula et al. 2011). This is consistent with opening and growth of the Meliata oceanic basin during Triassic period (e.g., Putiš et al. 2012). An extensional tectonic regime enabled activation of faults 
and fractures in Paleozoic metamorphic rocks, as well as circulation of hydrothermal fluids and solutions.

However, accessory xenotime, monazite, and apatite occur also in the quartz-tourmaline stage of stibnite and siderite ore veins of the Gemeric Superunit (Varček 1985). The chemical monazite $\mathrm{U}-\mathrm{Th}-\mathrm{Pb}$ ages from stibnite veins cluster around 130 and $80 \mathrm{Ma}$; the monazite dating from nearby Čučma, Klement vein yielded $120 \pm 9$ Ma and $76 \pm 12 \mathrm{Ma}$ (Hurai et al. 2008). Consequently, the hydrothermal system of the Gemeric Superunit was developed from Early Cretaceous crustal thickening to Late Cretaceous transpressive shearing and extension (Hurai et al. 2008). This is apparently younger than the early Alpine, Late Triassic REE-U mineralization studied here.

\subsection{Remarks on possible source and origin of REE-U mineralization}

The main, quartz-apatite-REE phosphates-uraninite vein mineralization near Čučma represents the best known example of hydrothermal REE-U accumulation in the Gemeric Superunit, as well as in the whole Western Carpathians. Similar smaller vein- and impregnationtype REE-U associations with monazite, xenotime, and uraninite occur in an analogous geological setting of the Lower Paleozoic basement of the Gelnica Group in broader vicinity of Čučma, especially in SW part of the Gemeric Superunit (Rojkovič et al. 1999).

Spatially, the REE-U mineralization is commonly related with the Spiš-Gemer granitic rocks. The granites represent specialized evolved S-type, tin-bearing suite, generally also enriched in $\mathrm{U}$ and fluxes (B, F and P) (e.g. Broska and Uher 2001). Consequently, these granites could have been an important source of U, REE, Y, as well as $\mathrm{P}$ and $\mathrm{F}$ for the studied mineralization. Boron- and F-bearing fluids most likely escaped from nearly granite intrusion to adjacent metamorphic rocks. The REE-U mineralization at Čučma does not contain B minerals but tourmaline-quartz dikes occur in the vicinity of the REE$\mathrm{U}$ vein and in adjacent B-rich granites. On the contrary, F-bearing fluids released from granitic rocks possibly contributed during precipitation of fluorapatite and associated minerals in the Čučma REE-U vein. However, the granites display Permian crystallization age (e.g., Poller et al. 2002) and consequently the REE-Y vein mineralization could not represent a direct magmatic hydrothermal derivate. On the other hand, the early Alpine, Late Triassic thermal and tectonic rejuvenation of the Gemeric Superunit indicated by tectonic and geochronologic data (Radvanec et al. 2009; Grecula et al. 2011), probably enabled some partial alteration and leaching of the Permian granites and adjacent Paleozoic metamorphic rocks and migration of hydrothermal F-rich fluids, enriched in P, REE, and U into suitable host-rock environment.
Uranium was deposited with REE phases as documented by the parallel bands of uraninite inclusions in botryoidal aggregates of xenotime-( $\mathrm{Y})$. We propose uraninite precipitation as a result of cyclic local $\mathrm{U}$ oversaturation in fluids on growing xenotime surface as indicated by textural evidence (Fig. 9). The second possible explanation of this assemblage and texture could be a partial fluid-driven dissolution-reprecipitation of U-bearing xenotime and formation of uraninite-rich bands by metasomatic alkali-rich fluids containing $U$ released from the altered xenotime. Such mechanism was proposed for similar thorite and uraninite inclusions in xenotime and monazite in Norwegian granitic pegmatites (Hetherington and Harlov 2008).

The attempts to estimate temperature conditions of primary minerals of the REE-U mineralization (quartz, fluorapatite, REE phosphate phases and uraninite) were unsuccessful. Fluid inclusions in quartz or apatite are very scarce and too small, and measured concentrations of Ti in quartz were under detection limit of electron microprobe (c. $10 \mathrm{ppm} \mathrm{Ti}$ in the special program file), which indicated temperature lower than $\sim 530^{\circ} \mathrm{C}$ according to Ti-in-quartz geothermometer (Wark and Watson 2006). On the basis of experimental data supported by natural low-pressure metapelite values, the miscibility of $\mathrm{Y}$ in monazite increases with temperature and pressure, from 3 to about 25 at. $\% \mathrm{Y}$ at 300 to $1000{ }^{\circ} \mathrm{C}$ and 2 to $15 \mathrm{kbar}$, but $\mathrm{Ce}$ concentrations in coexisting xenotime do not exceed 3 at. \% at analogous $P-T$ conditions (Gratz and Heinrich 1997; Andrehs and Heinrich 1998; Spear and Pyle 2002; Mogilevsky 2007). Our monazite and xenotime miscibility gap boundaries are roughly similar to the experimental data. The application of the monazitexenotime geothermometry, based on Y + HREE molar fraction in monazite (Gratz and Heinrich 1997; Spear and Pyle 2002; Mogilevsky 2007), reveals an equilibrium temperature, generally below $400{ }^{\circ} \mathrm{C}$, due to very low $\mathrm{Y}$ and HREE content in monazite from Čučma (up to 0.025 apfu). The extremely low Th content in the monazite also suggests growth under hydrothermal conditions (Schandl and Gorton 2004). Consequently, all these data indicate hydrothermal conditions for origin of primary minerals (quartz, fluorapatite, xenotime, monazite and uraninite), and precipitation of florencite-(Ce) and goyazite represented a younger, low-T replacement association.

Acknowledgements. This work was financially supported by the Slovak Research and Development Agency under the contract Nos APVV-0557-06, APVV-0279-07, APVV-0081-10, and VEGA Agency No. 1/0255/11, No. $1 / 0257 / 13$. This paper benefited from the constructive reviews by David Lenz and Igor Broska. Editorial handling by Jiří Sejkora and editor-in-chief Vojtěch Janoušek are highly appreciated. 


\section{References}

AmLi R, GRIFFIn W (1975) Microprobe analysis of REE minerals using empirical correction factors. Amer Miner 60: 599-606

Andrehs G, HeInRICH W (1998) Experimental determination of REE distributions between monazite and xenotime: potential for temperature-calibrated geochronology. Chem Geol 149: 83-96

Bagdasaryan GP, Cambel B, Veselský J, Gukasjan RCh (1977) $\mathrm{K}$-Ar ages of crystalline rock complexes of the Western Carpathians and preliminary interpretation of results. Geol Zbor Geol Carpath 28: 219-242 (in Russian)

Bajaník Š, Hanzel V, Ivanička J, Mello J, Pristaš J, ReichWAlder P, Snopko L, VozÁr J, VozÁRovÁ A (1983) Explanation to geological map of the Slovenské Rudohorie Mts. - eastern part. Dionýz Štúr State Geological Institute, Bratislava, pp 1-223 (in Slovak)

Bajaní Š, Hanzel V, IvaničKa J, Mello J, Pristaš J, ReichWALDER P, SNOPKo L, VozÁr J, VozÁrovÁA (1984) Geological map of the Slovenské Rudohorie Mts. - eastern part. Dionýz Štúr State Geological Institute, Bratislava (in Slovak)

Bowles JFW (1990) Age dating of individual grains of uraninite in rocks from electron microprobe analyses. Chem Geol 83: 47-53

Broska I, Uher P (2001) Whole-rock chemistry and genetic typology of the Western Carpathian, Variscan granites. Geol Carpath 52: 79-90

Broska I, Williams CT, Uher P, Konečný P, Leichmann J (2004) The geochemistry of phosphorus in different granite suites of the Western Carpathians, Slovakia: the role of apatite and P-bearing feldspar. Chem Geol 205: 1-15

Cambel B, Král J, Burchart J (1990) Isotopic Geochronology of the Western Carpathian Crystalline Complex with Catalogue of Data. Veda, Bratislava, pp 1-184 (in Slovak)

CAMERON-Schiman M (1978) Electron microprobe study of uranium minerals and its application to some Canadian deposits. Unpublished Ph.D. Thesis, University of Alberta, Edmonton, pp 1-686

Demko R, Ferenc Š, Biroñ A (2011) Synthesis of mineralogical and petrographical research in Košice, Kurišková deposit (years 2006 to 2010). Unpublished report, pp 1-92 (in Slovak)

Demko R, Ferenc Š, Biroň A, Novotný L, Bartalský B (2012) The genesis of Kurišková U-Mo ore deposit. Esemestník 1: 24-25

FARYAD SW (1991) Metamorphism of the Early Paleozoic sedimentary rocks in the Gemericum. Miner Slov 23: 315-324

GIERÉ R (1996) Formation of rare earth minerals in hydrothermal systems. In: JonES AP, Wall F, Williams CT (eds) Rare Earth Minerals: Chemistry, Origin and Ore Deposits. Chapman \& Hall, London, pp 105-150

Gratz R, Heinrich W (1997) Monazite-xenotime thermobarometry: experimental calibration of the miscibility gap in the binary system $\mathrm{CePO}_{4}-\mathrm{YPO}_{4}$. Amer Miner 82: $772-780$

Grecula P, Kobulský J, GazdačKo L', Németh Z, HrašKo L', Novotný L, Maglay J (2009) Geological map of the Spiš-Gemer Ore Mts. 1 : 50 000. Dionýz Štúr State Geological Institute, Bratislava

Grecula P, Kobulský J, GazdačKo L', Németh Z, HrašKo L', Novotný L, Maglay J, Pramuka S, Radvanec M, KuCharič L, Bajtoš P, ZÁHorová L' (2011) Explanations to geological map of the Spiš-Gemer Ore Mts. Dionýz Štúr State Geological Institute, Bratislava, pp 1-308 (in Slovak)

Hetherington CJ, Harlov DE (2008) Metasomatic thorite and uraninite inclusions in xenotime and monazite from granitic pegmatites, Hidra anorthosite massif, southwestern Norway: mechanics and fluid chemistry. Amer Miner 93: 806-820

Hurai V, Lexa O, Schulmann K, Montigny R, Prochaska W, Frank W, KoneČnÝ P, Král J, Thomas R, Chovan M (2008) Mobilization of ore fluids during Alpine metamorphism: evidence from hydrothermal veins in the Variscan basement of Western Carpathians, Slovakia. Geofluids 8: 181-207

IVANičKa J, Snopko L, Snopková P, VozÁRovÁ A (1989) Gelnica Group - Lower Unit of Spišsko-Gemerské Rudohorie Mts. (Early Paleozoic, West Carpathians). Geol Zbor Geol Carpath 40: 483-501

JANeCZeK J, EwIng RC (1996) Florencite-(La) with fissiogenic REEs from a natural fission reactor at Bangombé, Gabon. Amer Miner 81: 1263-1269

Koнút M, Stein H (2005) Re-Os molybdenite dating of granite-related $\mathrm{Sn}-\mathrm{W}-\mathrm{Mo}$ mineralization at Hnilec, Gemeric Superunit, Slovakia. Mineral Petrol 85: 117-129

Kohút M, Trubač J, Novotný L, Ackerman L, Demko R, Bartalský B, Erban V (2013) Geology and Re-Os molybdenite geochronology of the Kurišková U-Mo deposit (Western Carpathians, Slovakia). J Geosci 58: 271-282

KoneČnÝ P, Siman P, Holický I, Janák M, Kollárová $\mathrm{V}$ (2004) Method of monazite dating by means of the electron microprobe. Miner Slov 36: 225-235 (in Slovak)

Mogilevsky P (2007) On the miscibility gap in monazitexenotime systems. Phys Chem Miner 34: 201-214

MöLler P (1989) Rare earth mineral deposits and their industrial importance. In: MÖller P, ČERNÝ P, SAUPÉ F (eds) Lanthanides, Tantalum and Niobium. Springer-Verlag, Berlin, pp 171-188

Montel J-M, Foret S, Veschambre M, Nicollet C, Provost A (1996) Electron microprobe dating of monazite. Chem Geol 131: 37-53

Petrík I, Kohút M (1997) The evolution of granitoid magmatism during the Hercynian orogen in the Western Carpathians. In: Grecula P, Hovorka D, Putiš M (eds) Geological Evolution of the Western Carpathians. Miner Slov - Monograph, pp 235-252 
Plášil J, SeJKora J, Č́tKa J, ŠKoda R, Goliáš V. (2009) Supergene mineralization of the Medvědín uranium deposit, Krkonoše Mountains, Czech Republic. J Geosci 54: 15-56

Poller U, Uher P, Broska I, Plašienka D, Janák M (2002) First Permian-Early Triassic zircon ages for tin-bearing granites from the Gemeric unit (Western Carpathians, Slovakia): connection to the post-collisional extension of the Variscan orogen and S-type granite magmatism. Terra Nova 14: 41-48

Putiš M, Sergeev S, Ondrejka M, Larionov A, Siman P, SPIŠIAK J, UhER P, PADERIN I (2008) Cambrian-Ordovician metaigneous rocks associated with Cadomian fragments in the West-Carpathian basement dated by SHRIMP on zircons: a record the Gondwana active margin setting. Geol Carpath 59: 3-18

Putiš M, Koppa M, Snárska B, Koller F, Uher P (2012) The blueschist-associated perovskite-andradite-bearing serpentinized harzburgite from Dobšiná (the Meliata Unit), Slovakia. J Geosci 57: 221-240

Radvanec M, Konečný P, Ondrejka M, Putiš M, Uher P, NÉmeth Z (2009) The Gemeric granites as an indicator of the crustal extension above the Late-Variscan subduction zone and during the Early Alpine riftogenesis (Western Carpathians): an interpretation from the monazite and zircon ages dated by CHIME and SHRIMP methods. Miner Slov 41: 381-394

RANCHIN G (1968) Contribution à l' étude de la répartition de l' uranium à l' état de traces dans les roches granitiques saines les uranites à teneur élevée du Massif de SaintSylvestre (Limousin - Massif Central Français). Sci de la Terre 13: 161-205

RoJkovič I (1993) Minerals of the crandallite series in quartz-apatite vein near Čučma. Miner Slov 25: 151-153 (in Slovak)

RoJKovič I (1997) Uranium mineralization in Slovakia. Acta Geol Univ Comen Monogr Ser., pp 1-117

Rojkovič I, KoneČný P, Novotný L, PušKelová L, StrešKo V (1999) Quartz-apatite-REE vein mineralization in Early Paleozoic rocks of the Gemeric Superunit, Slovakia. Geol Carpath 50: 215-227

SAMSON IM, Wood SA (2005) The rare-earth elements: behaviour in hydrothermal fluids and concentration in hydrothermal mineral deposits, exclusive of peralkaline settings. In: LiNNEN RL, SAMSON IM (eds) Rare-Element Geochemistry and Ore Deposits. Geological Association of Canada, Short Course Notes 17: 406-452

SchandL ES, Gorton MP (2004) A textural and geochemical guide to the identification of hydrothermal monazite; criteria for selection of samples for dating epigenetic hydrothermal ore deposits. Econ Geol 99: 1027-1035

SNOPKO L (1967) Lithological characteristics of the Gelnica Series. Záp Karpaty Sér Geol 7: 103-152 (in Slovak)

SNOPKOVÁ P, SNOPKO L (1979) Biostratigraphy of Gelnica Series of Spišsko-Gemerské Rudohorie Mts. on the basis of palynological study. Záp Karpaty Sér Geol 19: 57-102 (in Slovak)

SotÁK J, VozÁRovÁ A, IvaniČKa J (1999) New microfossils from the Early Paleozoic of the Gemericum. Geol Carpath 50: 72-74

Spear FS, Pyle JM (2002) Apatite, monazite and xenotime in metamorphic rocks. In: KoHN MJ, RAKOvan J, HugheS JM (eds) Phosphates: Geochemical, Geobiological and Materials Importance. Mineralogical Society of America and Geochemical Society Reviews in Mineralogy and Geochemistry 48: 293-335

ŠváB J, Tulis J, BADÁR J (1966) Final report about results of geological survey in Čučma locality. Unpublished Manuscript, Dionýz Štúr State Geological Institute, Bratislava, pp 1-113 (in Slovak)

TRÉGER M (1973) Occurrences of uranium-bearing phosphates in the Spišsko-Gemerské Rudohorie Mts. Miner Slov 5: 61-64 (in Slovak)

Uher P, ŠTeVko M (2009) Apatite-REE-uranium mineralization in Čučma near Rožňava. Minerál 17: 415-419 (in Slovak)

VARČEK C (1977) Some rare type of mineralization in the Spišsko-Gemerské Rudohorie Mountains. In: Ložiskotvorné procesy Západných Karpát. PriF UK, Bratislava, pp 93-99 (in Slovak)

VARČEK C (1985) Metallogenetic characteristics of the Spišsko-Gemerské Rudohorie Mountains and the position of the Rudňany ore field. In: CAMBEL B, JARKOVSKÝ J (eds) The Rudňany Ore Field: Geochemical and Metallogenetic Characteristics. Veda, Bratislava, pp 61-77 (in Slovak)

VozÁrovÁ A (1993) Variscan metamorphism and crustal evolution of the Gemericum. Záp Karpaty Sér Min Petr Metalog Geoch 16: 55-117 (in Slovak)

VozÁrováA, SotÁK J, IVANIČKa J (1998) A new microfauna from the Early Paleozoic of the Gemericum (Foraminifera): constrains for another fossils or subfossils. In: RaKús M (ed) Geodynamic Development of the Western Carpathians. Dionýz Štúr Publishing, Bratislava, pp 63-74

VozÁrováA, Šarinová K., Sergeev S, Larionov A, PresnyaKOV S (2010) Late Cambrian/Ordovician magmatic arc type volcanism in the Southern Gemericum basement, Western Carpathians, Slovakia: U-Pb (SHRIMP) data from zircons. Int J Earth Sci 99: 17-37

VozÁrová A, Šmelko M, Paderin I, Larionov A (2012) Permian volcanics in the Northern Gemericum and Bôrka Nappe system: U-Pb zircon dating and the implications for geodynamic evolution (Western Carpathians, Slovakia). Geol Carpath 63: 191-200

WARK DA, WATSON EB (2006) TitaniQ: a titanium-in-quartz geothermometer. Contrib Mineral Petrol 152: 743-754

Yongliang X, Yusheng Z (1991) The mobility of rare-earth elements during hydrothermal activity: a review. Chinese J Geochem 10: 296-306 